\title{
Systematic Literature Review and Expert Opinion for the Use of Viscosupplementation with Hyaluronic Acid in Different Localizations of Osteoarthritis
}

\begin{abstract}
Alberto Migliore, (DD' Gianfranco Gigliucci, (D)' Lyudmila Alekseeva, ${ }^{2}$ Raveendhara R Bannuru, (D) ${ }^{3}$ Tomasz Blicharski, ${ }^{4}$ Demirhan Diracoglu, ${ }^{5}$ Athanasios Georgiadis, ${ }^{6}$ Hesham Hamoud, ${ }^{7}$ Natalia Martusevich, (iD ${ }^{8}$ Marco Matucci Cerinic, 'Jan Perduk, ${ }^{10}$ Imre Szerb, "' Tomáš Trč, ${ }^{12}$ Xavier Chevalier $^{13}$

'Rheumatology Unit - San Pietro Fatebenefratelli Hospital, Rome, Italy;

${ }^{2}$ Department of Metabolic Diseases of Bone and Joints, V.A. Nasonova Research Institute of Rheumatology, Moscow, Russian Federation; ${ }^{3}$ Center for Treatment Comparison and Integrative Analysis (CTCIA) at Tufts Medical Center, Boston, MA, USA; ${ }^{4}$ Lubelskie Centrum Diagnostyczne, Świdnik, Poland; ${ }^{5}$ Department of Physical Medicine and Rehabilitation, Istanbul University, Istanbul, Turkey; ${ }^{6}$ Department of Rheumatology, University of loannina, loannina, Greece; ${ }^{7}$ Department of Rheumatology Al Azhar University, Cairo, Egypt; ${ }^{8}$ Department of Rheumatology, Belarusian State Medical University, Minsk, Belarus; 'Division of Rheumatology, University of Florence, Florence, Italy; ${ }^{10}$ Department of

Musculoskeletal Medicine and Sports, Hospital Košice -Šaca, Kosice Saca, Slovakia;

"Semmelweis University, Department of

Traumatology Uzsoki Hospital, Department of Orthopaedics\&Traumatology FIFA Medical Centre of Excellence Uzsoki u. 29-4I, Budapest, Hungary; '2 Department of Orthopaedic Surgery, Charles University and Motol University Hospital, Prague, Czech Republic; ${ }^{13}$ Rheumatology Division, Henri-Mondor Hospital, Créteil, France
\end{abstract}

Correspondence: Alberto Migliore Rheumatology Unit - San Pietro Fatebenefratelli Hospital, Rome, Italy Email reumafbf@libero.it

\begin{abstract}
Osteoarthritis (OA) is a significant cause of disability. Considering the increasing diffusion of the viscosupplementation (VS) with hyaluronic acid (HA), the International Symposium Intra Articular Treatment (ISIAT) appointed a Technical Expert Panel (TEP) to identify the criteria for successful VS with a specific HA in OA; this through a systematic literature review (SLR), performed following the PRISMA guidelines interrogating Medline, Embase, Cochrane Library, Cumulative Index to Nursing and Allied Health Literature (CINAHL), Grey Matters and American College of Rheumatology (ACR/EULAR) databases and the opinion of international experts. The research included only studies on adults and humans without limitations of language or time of publication. Researchers extracted both quantitative and qualitative data from each study. Mixed Methods Appraisal Tool (MMAT) was used to perform quality analysis for the level of evidence. The SLR retrieved 385 papers, 25 of which were suitable for the analysis. The TEP focused on the different formulations of the product Sinovial ${ }^{\circledR}$ [HA 0.8\%, HA 1.6\%, HA 2\%, 800-1200 kDa, HA 3.2\% (1400-2100 $\mathrm{kDa} / 65-110 \mathrm{kDa})]$. The choice was due to the vast amount of evidence available. The TEP weighed the evidence in two rounds of a Delphi survey; the results, and any disagreement, were discussed in a final session. Three domains were considered: 1) the patients' characteristics associated with the best results; 2) the contraindications and the conditions linked to increased risk of failure; 3) the clinical conditions in which VS is considered appropriate. The TEP concluded that VS with HA is safe and effective in the treatment of knee and hip OA of grades I to III and that it is possible to undertake VS in other situations (eg grade IV Kellgren-Lawrence - KL); a comprehensive examination of the patient should be performed before the procedure.
\end{abstract}

Keywords: appropriateness, osteoarthritis, personalized medicine, viscosupplementation

\section{Introduction}

Osteoarthritis (OA) is the most common disabling arthropathy, impairing the quality of life of patients and imposing a heavy burden on healthcare systems. Hand, knee, hip and spinal facets are the most affected joints, with an increasing prevalence with the age of the patients. Women are more affected than men, especially for hand and knee OA. Hunter and Bierma-Zeinstra ${ }^{1}$ found a prevalence between $10 \%$ and $30 \%$ of the population according to sex and the joint affected. The incidence of OA increases steeply after the age of 50 with a peak around 75 years. ${ }^{1}$ Roddy and Menz ${ }^{2}$ reported similar results in their review on foot OA. Peyron and Balazs ${ }^{3}$ first proposed viscosupplementation (VS) with Intraarticular Hyaluronic Acid (IAHA). The 
rationale is based on the mechanical and physiological properties of HA, an essential component of the joint tissues and the synovial fluid. Despite the large body of evidence on the safety and efficacy of VS-IAHA, this procedure is still an object of controversial by international guidelines. ${ }^{4}$ Osteoarthritis Research Society International (OARSI) ${ }^{5}$ and European League of Associations for Rheumatology $(\text { EULAR })^{6}$ guidelines granted IAHA a low-grade recommendation, while the National Collaborating Centre for Chronic Condition (NCC-CC) ${ }^{7}$ and American Academy of Orthopedic Surgeons (AAOS) ${ }^{8}$ guidelines recommended against IAHA. The AAOS guideline was criticized by Santilli et $\mathrm{al}^{9}$ who pointed out that the guideline was lacking multidisciplinarity being prepared mainly by orthopedic surgeons. Other aspects of the above guidelines raised objections: the difference between the highly selected patients enrolled in Randomized Controlled Trials (RCTs), reviewed in the guidelines, and patients in clinical practice; the selection of comparators; the limits of the criterion of the Minimum Clinically Important Improvement (MCII) and the underestimation of the clinical relevance of differences in phenotypes of OA. ${ }^{10}$ Maheu et al maintained that OA should be considered as a multifactorial disease requiring a flexible approach and an accurate definition of the most suitable patients for VS. ${ }^{11}$ The European Society for Clinical and Economic Aspects of Osteoporosis, Osteoarthritis and Musculoskeletal Diseases (ESCEO) ${ }^{12}$ guideline recommended a multi-step approach to OA, starting with measures like weight reduction, aerobic exercise, physiotherapy and rehabilitation, followed by Symptomatic Slow-Acting Drugs for Osteoarthritis (SySADOAs), topical or oral Non-Steroidal Anti-Inflammatory Drugs (NSAIDs), duloxetine or analgesics. The guideline defined VS as an effective treatment for knee OA, with a positive impact on pain and function and as the last step before surgery. Finally, the EUROpean VIScosupplementation Consensus group (EUROVISCO) ${ }^{13}$ issued detailed recommendations for a proper selection of the patients to be treated with IAHA. The International Symposium Intra Articular Treatment (ISIAT), which gathers leading experts on IA treatment every two years, appointed a multinational, multidisciplinary Technical Expert Panel (TEP), composed by the authors of this paper, to identify predictors of a positive or negative outcome of VS with IAHA. The TEP focused on the different formulations of a single product: Sinovial ${ }^{\circledR}$ (other brand names: Intragel ${ }^{\circledR}$ - Gelsyn- $3^{\circledR}$ ) [HA $0.8 \%$, HA $1.6 \%$, HA $2 \%, 800-1200 \mathrm{kDa}$, HA $3.2 \%$ (1400-2100 kDa/65-110 kDa)]. The choice was made for four main reasons: the large body of available scientific evidence; the extensive use of the product worldwide; the well-known safety profile and the direct experience of the TEP members. This paper reports the results of the opinion of the TEP to improve appropriate use of VS with HA in clinical practice.

\section{Materials and Methods}

The TEP paid great attention to achieving consensus through rigorous scientific methodology. A Systematic Literature Review (SLR) was conducted by a librarian and the papers retrieved were analyzed and weighed by a data extraction form. The steering committee of the TEP (A. Migliore-Scientific Coordinator \& Steering Committee, R. Bannuru, X. Chevalier, D. Diracoglou, C. Matucci-Steering Committees) selected 19 statements that were discussed in a first plenary meeting (Lisbon November 2019). During the meeting, the TEP defined 21 statements that were weighed and discussed in two rounds of a Delphi survey; the results, including any disagreements, were further discussed in a final plenary session (Lugano February 2020) where final agreement was reached on the statements.

\section{Systematic Literature Research}

The SLR explored the use of HA in all joints affected by $\mathrm{OA}$ to identify predictors of a positive or negative clinical response. The steering committee of the TEP identified the clinical questions for the SLR, formulated in the Patient, Problem or Population/Intervention/ Comparison, control or comparator/Outcome(s) (PICO) [Timing, duration or date of publication (T)/Study type (S)] format. Table 1 shows the structure of the PICO research.

The following databases were interrogated: Medline, Embase, Cochrane Library, Cumulative Index to Nursing and Allied Health Literature (CINAHL), Grey Matters (http://www.cadth.ca/), and American College of Rheumatology (ACR)/EULAR abstracts. The research

Table I Formulation of Clinical Questions in PICO Format

\begin{tabular}{|l|l|}
\hline P & Human Patients, Adults, Osteoarthritis \\
I & Hyaluronic acid, infiltrations, viscosupplementation or hyaluronic \\
& acid $0.8 \%, 1.6 \%, 2 \%, 800-1200 \mathrm{kDa}, 3.2 \%(1400-2100 \mathrm{kDa} / 65-$ \\
& $110 \mathrm{kDa})$, infiltrations, viscosupplementation \\
C & - \\
O & All \\
\hline
\end{tabular}


included only studies on adults, humans, without limitations of language or time of publication, this to minimize any risk of bias due to the above factors. An ad hoc extraction module collected the data from the individual studies. The extraction module considered: author, year, publication type, study design and structure, inclusion/exclusion criteria, number of participants, the definition of OA, type of HA formulation [HA $0.8 \%$, HA $1.6 \%$, HA $2 \%, 800-1200 \mathrm{kDa}$, HA $3.2 \%(1400$ $2100 \mathrm{kDa} / 65-110 \mathrm{kDa})$ ], a summary of findings. The studies retrieved were reviewed by the panelists who selected a subgroup of studies that were considered worthy of further assessment. Both quantitative and qualitative synthesis of data was performed (Figure 1). The characteristics of the populations enrolled in the selected studies were analyzed with a special focus on patients' subgroups displaying a high or low response rate to VS. Two experts provided each an independent revision of the study: any disagreements were submitted to a third expert. The quality of the selected papers was assessed by two independent experts using the 2018 version of the Mixed Method Appraisal Tool (MMAT); the appropriateness of study aim, methodology, design, data collection and analysis, and presentation of findings were evaluated. ${ }^{14}$

\section{Results}

After exclusion of duplicates, the SLR retrieved 385 studies, 25 of which were considered suitable for analysis. The studies were first examined for the exclusion and inclusion criteria. The process of SLR, according to the Preferred Reporting Items for Systematic reviews and Meta-Analyses (PRISMA) statement ${ }^{15}$ with the number of studies retrieved, excluded, and selected is shown in Figure 1 . The quality of the included studies was critically evaluated using the MMAT with scores summarized in Table 2. Tables 3 and 4 summarize the main features of the selected studies.

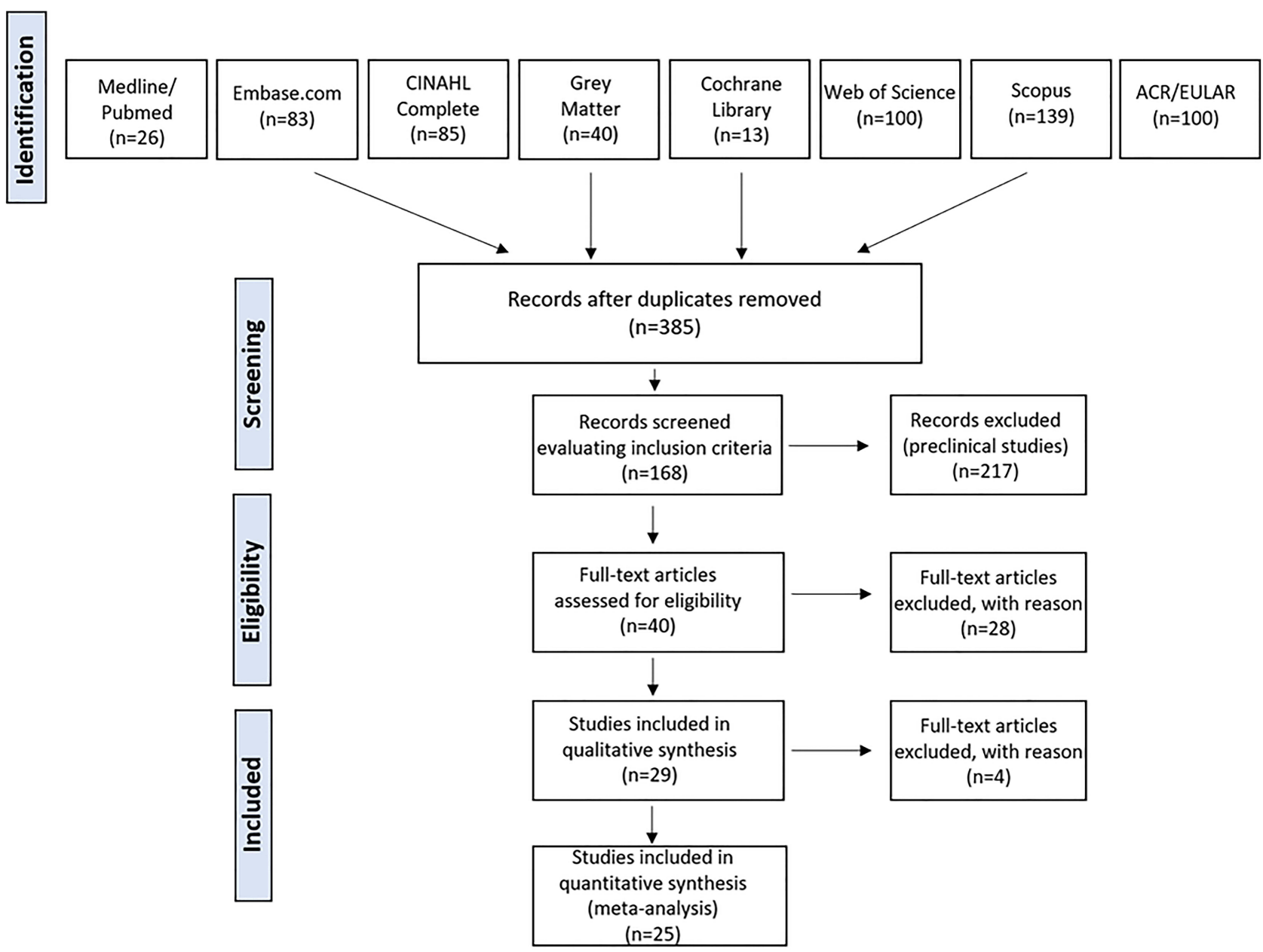

Figure I Selection of papers during the SLR. 
Table 2 Calculated MMAT Scores for Studies Included in This Systematic Review

\begin{tabular}{|c|c|}
\hline Study & MMAT Score \\
\hline Castellacci $2004^{16}$ & 4 \\
\hline Depont $2004^{46}$ & 3 \\
\hline Theiler $2005^{17}$ & 5 \\
\hline Pavelka $20 \mathrm{II}^{22}$ & 5 \\
\hline Polacco $2013^{18}$ & 5 \\
\hline Bottegoni $2014^{20}$ & 5 \\
\hline Papalia $2016^{19}$ & 4 \\
\hline Abate $2017^{25}$ & 5 \\
\hline Papalia $2017^{21}$ & 4 \\
\hline Galluccio $2021^{47}$ & 5 \\
\hline Manciameli $2018^{35}$ & 4 \\
\hline Migliore $2018^{48}$ & 5 \\
\hline Papalia $2019^{23}$ & 5 \\
\hline Migliore $2012^{24}$ & 5 \\
\hline Lùrati $2015^{32}$ & 3 \\
\hline Abate and Salini $2017^{25}$ & 5 \\
\hline Abate $2014^{26}$ & 5 \\
\hline La Paglia $2017^{27}$ & 5 \\
\hline Busilacchi 20II ${ }^{29}$ & 3 \\
\hline Gigante $2013^{28}$ & 5 \\
\hline D'Avola $2013^{34}$ & 3 \\
\hline Bartoloni $2019^{33}$ & 4 \\
\hline Tenti $2017^{31}$ & 5 \\
\hline Callegari $20 \mathrm{II}^{36}$ & 5 \\
\hline Roux $2007^{30}$ & 5 \\
\hline
\end{tabular}

\section{Analysis of the Inclusion/Exclusion Criteria}

The analysis of the inclusion/exclusion criteria for each OA localization was the basis for the generation of the statements, which were subjected to two rounds of the survey using the Delphi method. In analyzing the exclusion criteria, the TEP considered that some of them (eg localization and concomitant pathologies) were intended to select the most suitable patients. In contrast, others (eg pharmacological treatments and previous VS procedures) were aimed at allowing a correct evaluation of efficacy. The TEP considered separately knee OA and OA in other joints. The inclusion/exclusion criteria reported in most relevant studies, or recurring more frequently, are commented below; additional criteria are provided in the above Tables 3 and 4 .

\section{Knee OA}

\section{Inclusion Criteria}

Age between 18 and 82 years and symptomatic knee OA confirmed by X-rays were the inclusion criteria defined by Castellacci. ${ }^{16}$ Theiler ${ }^{17}$ considered age between 18 and 85 years; pain on walking $>30 \mathrm{~mm}$ measured by a $100 \mathrm{~mm}$ Visual Analogue Scale (VAS). Polacco ${ }^{18}$ included patients with symptomatic knee OA with a Western Ontario Mc Master University (WOMAC) score between 0 and 15 (mean 8.8) and with OA of Kellgren \& Lawrence (KL) grades from II to III. As for concomitant administration/ comparison to Platelet Rich Plasma (PRP), Papalia ${ }^{19}$ compared HA 3.2\% with PRP in a particular set of patients (professional soccer players) with lower age, from 34 to 39. Bottegoni ${ }^{20}$ included patients above 65 years only. Another study by Papalia ${ }^{21}$ considered patients with symptoms lasting for more than three months and with OA of $\mathrm{KL}$ grades I to III.

\section{Exclusion Criteria}

Castellacci ${ }^{16}$ excluded patients with local or systemic inflammatory conditions; Theiler ${ }^{17}$ considered exclusion criteria chondrocalcinosis, gout, crystal arthropathy, excessive joint effusion, severe axis deviation or other inflammatory diseases (Paget disease, history of knee surgery or intraarticular steroid injection in the last three months, chronic assumption of steroids). BMI $>32$, secondary $\mathrm{OA}$ and $\mathrm{KL}$ grade IV hip OA were among the exclusion criteria defined by Pavelka. ${ }^{22}$ For the evaluation of efficacy, the most common exclusion criteria were the use of systemic steroids and SySADOAs in the last three months and VS and treatment with IA steroids before enrollment. ${ }^{17,18,20,22}$ The TEP also considered studies comparing HA with PRP or association of HA with PRP. Such studies excluded patients below 45 or above 75 years. Additional exclusion criteria were medical conditions contraindicating the use of PRP, especially alterations of coagulation or immunodepression (see Table 2). ${ }^{19,23}$

\section{Other Joints}

Inclusion Criteria

For hip OA, a cohort study of 114 patients included patients aged $>40$ years, with symptomatic hip OA according to the American College of Rheumatology (ACR) criteria, and $\mathrm{KL}$ grades II or III. $^{24}$ Abate and Salini ${ }^{25}$ selected patients with KL II and III, clinically symptomatic for more than three months. For hip impingement, the pain was the most common inclusion criterion. ${ }^{26,27}$ For shoulder localizations, Gigante ${ }^{28}$ included patients aged between 35 and 70 years, affected by shoulder impingements of various degrees from bursitis to eccentric OA. In the study by Busilacchi, ${ }^{29}$ the inclusion criteria allowed patients with cuff pathology of grades I-IV of Neer's 


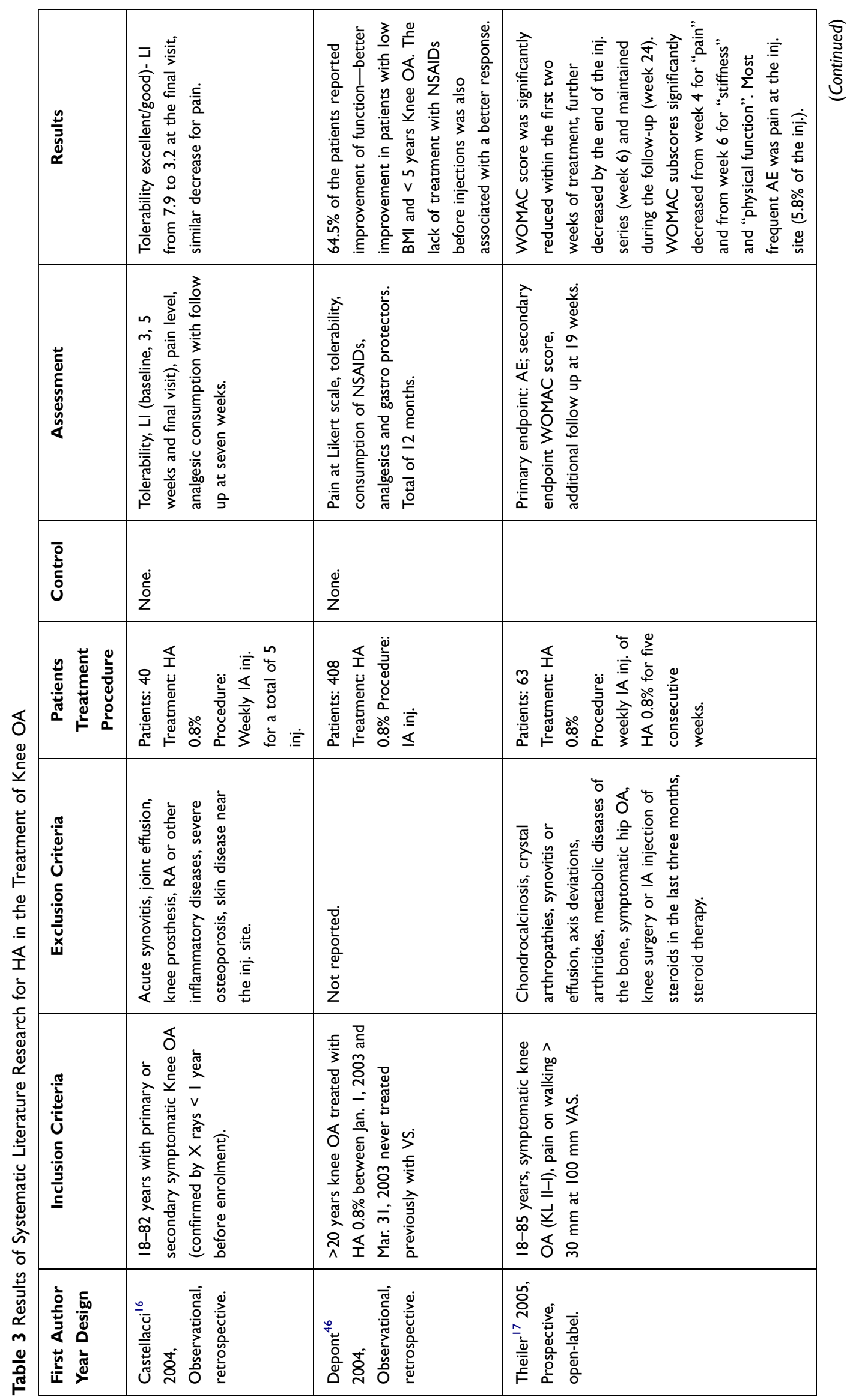




\begin{tabular}{|c|c|c|c|c|}
\hline $\begin{array}{l}\frac{\tilde{y}}{\vec{J}} \\
\underline{\vec{y}} \\
\ddot{x}\end{array}$ & 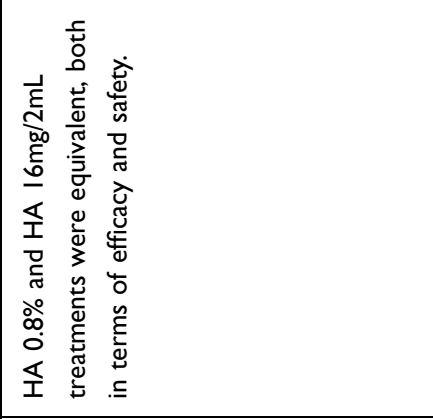 & 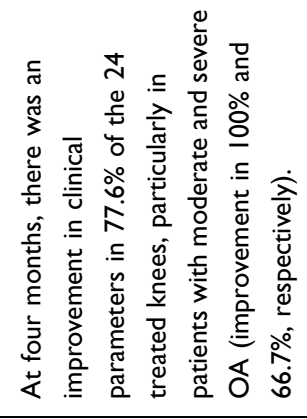 & 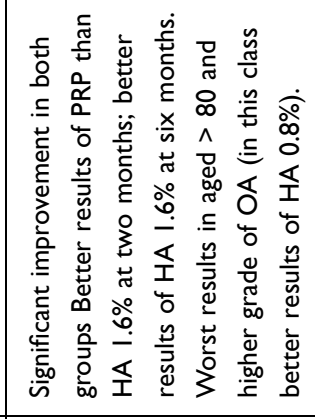 & 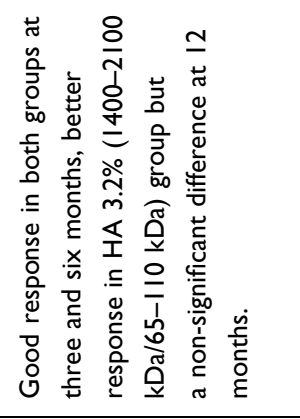 \\
\hline & 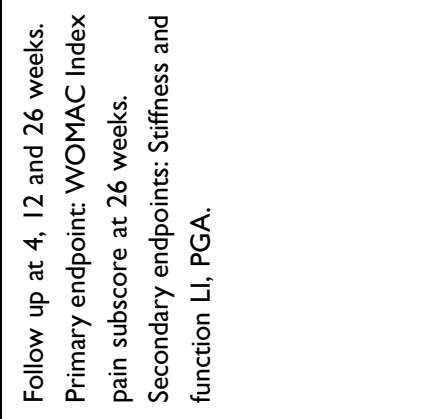 & 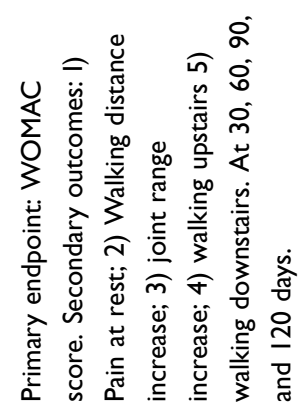 & 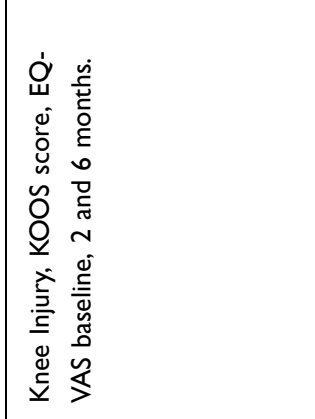 & 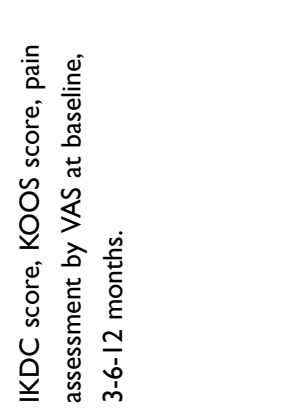 \\
\hline 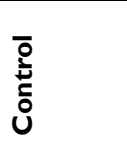 & 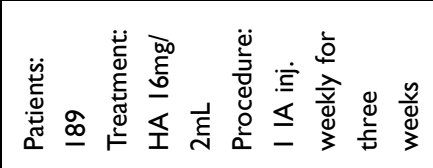 & $\begin{array}{l}0 \\
\stackrel{0}{Z} \\
z\end{array}$ & 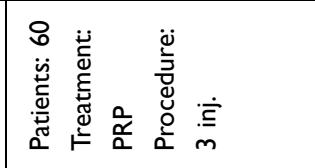 & 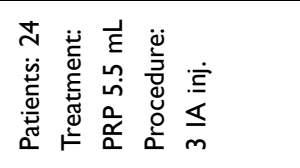 \\
\hline 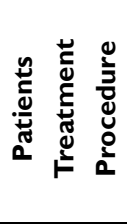 & 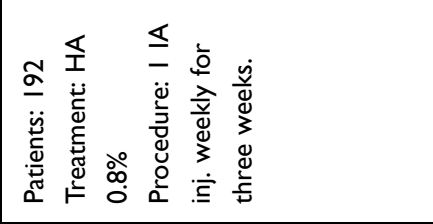 & 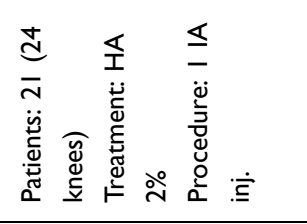 & 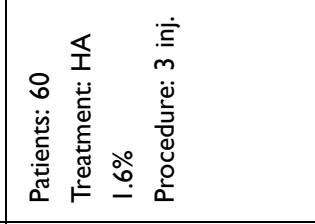 & 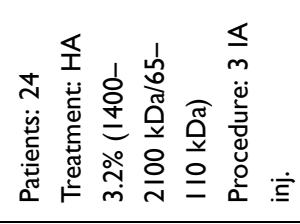 \\
\hline 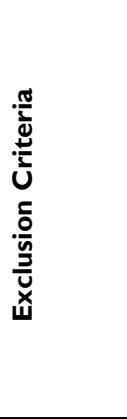 & 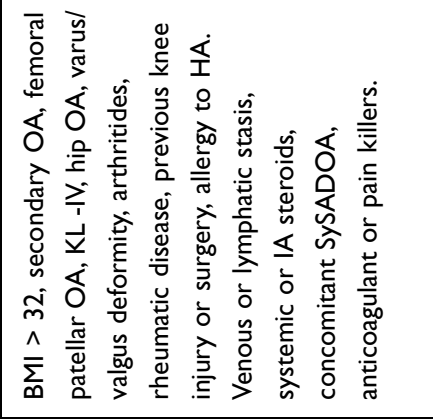 & 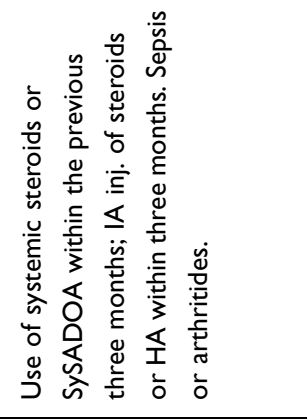 & 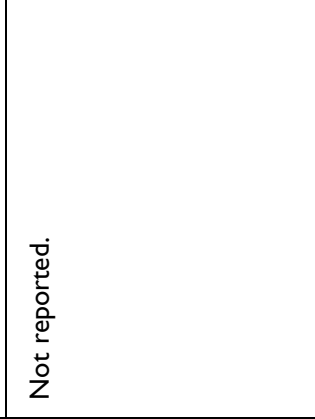 & 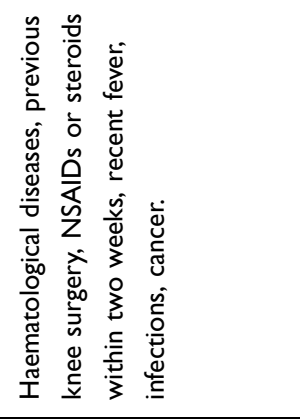 \\
\hline 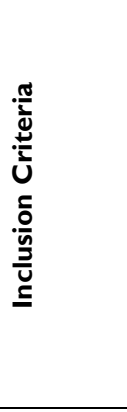 & 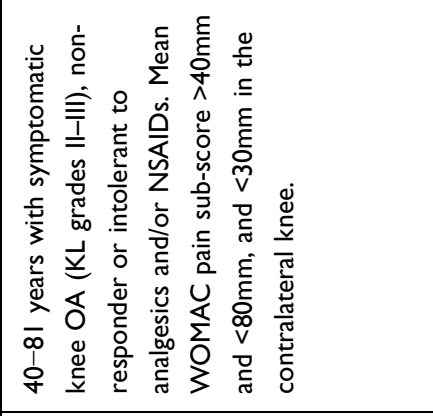 & 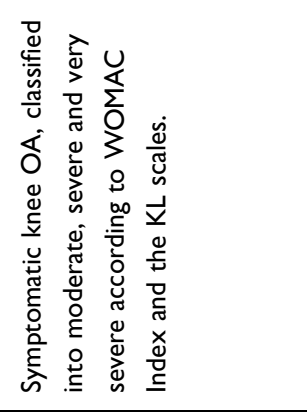 & 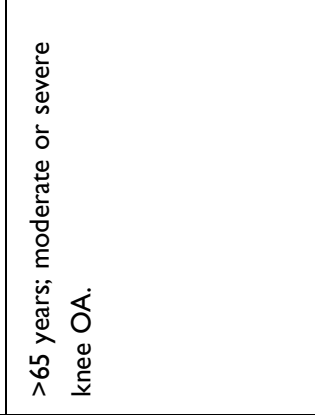 & 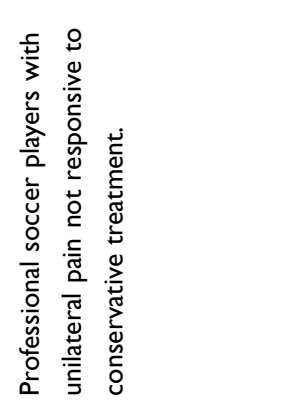 \\
\hline 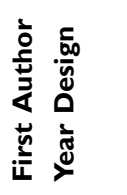 & 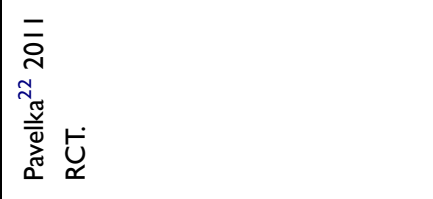 & 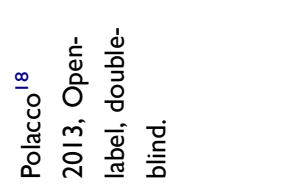 & 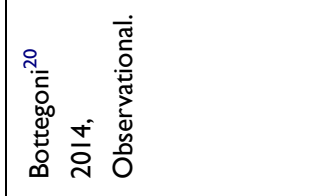 & 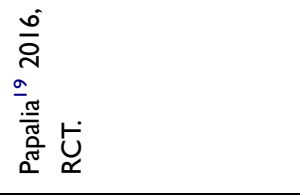 \\
\hline
\end{tabular}




\begin{tabular}{|c|c|c|}
\hline 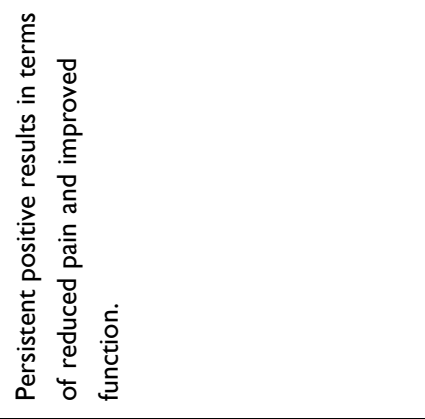 & 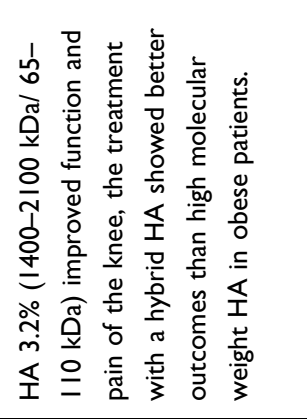 & 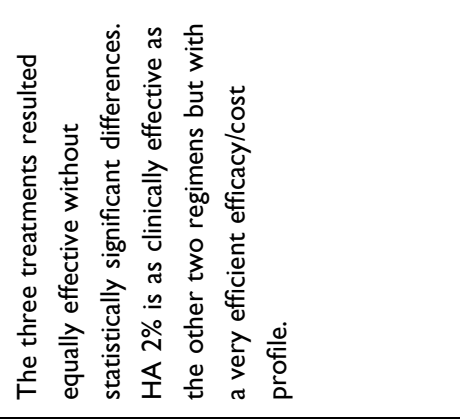 \\
\hline 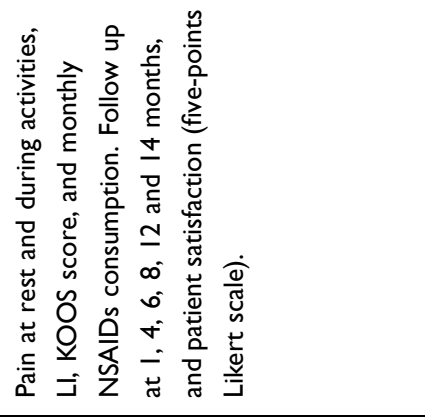 & 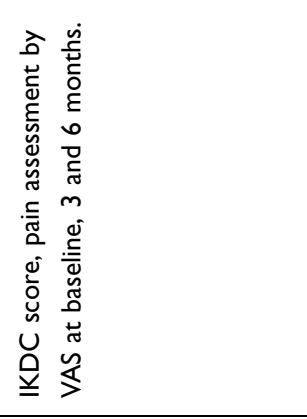 & 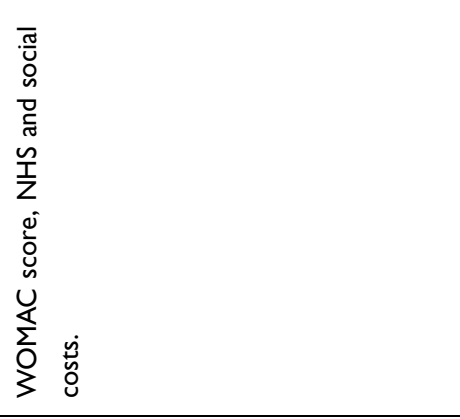 \\
\hline $\begin{array}{l}\text { ¿̇ } \\
\text { ż } \\
\end{array}$ & 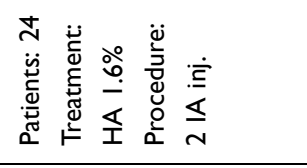 & 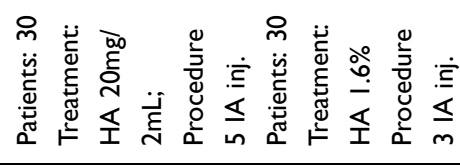 \\
\hline 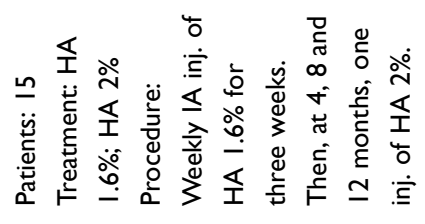 & 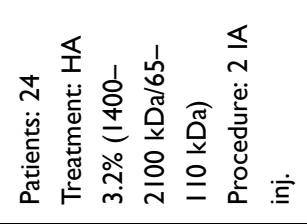 & 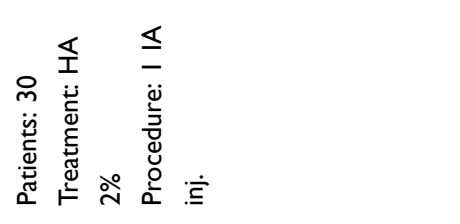 \\
\hline 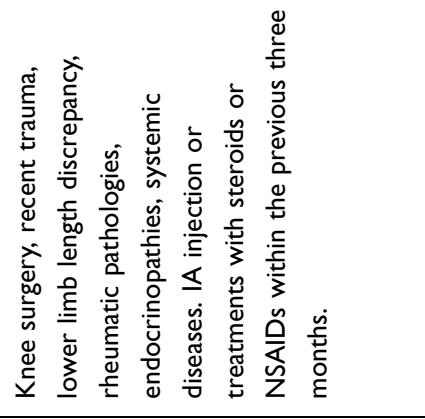 & 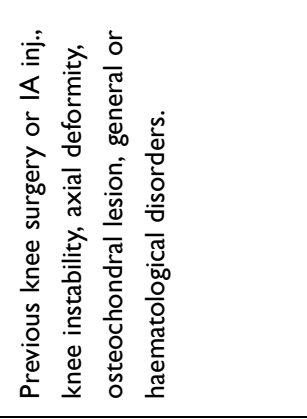 & 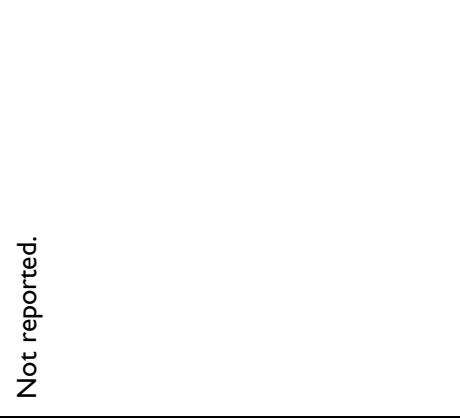 \\
\hline 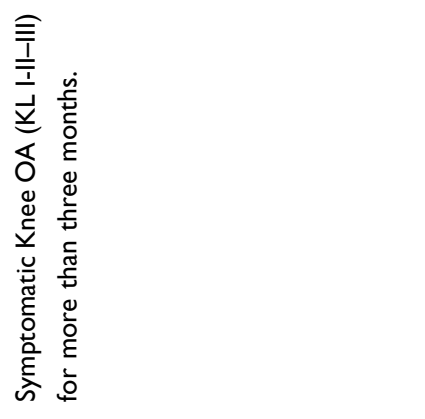 & 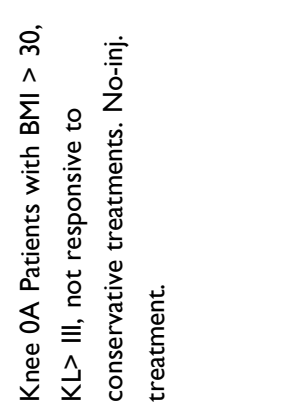 & 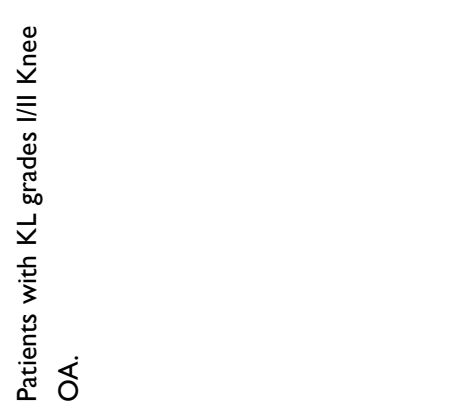 \\
\hline 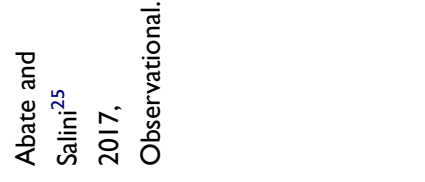 & 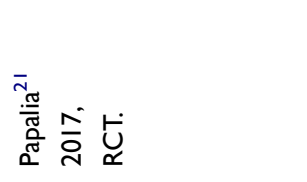 & 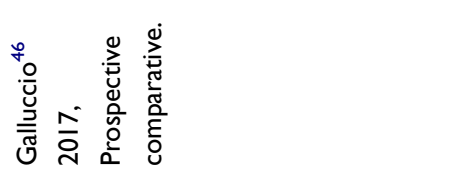 \\
\hline
\end{tabular}




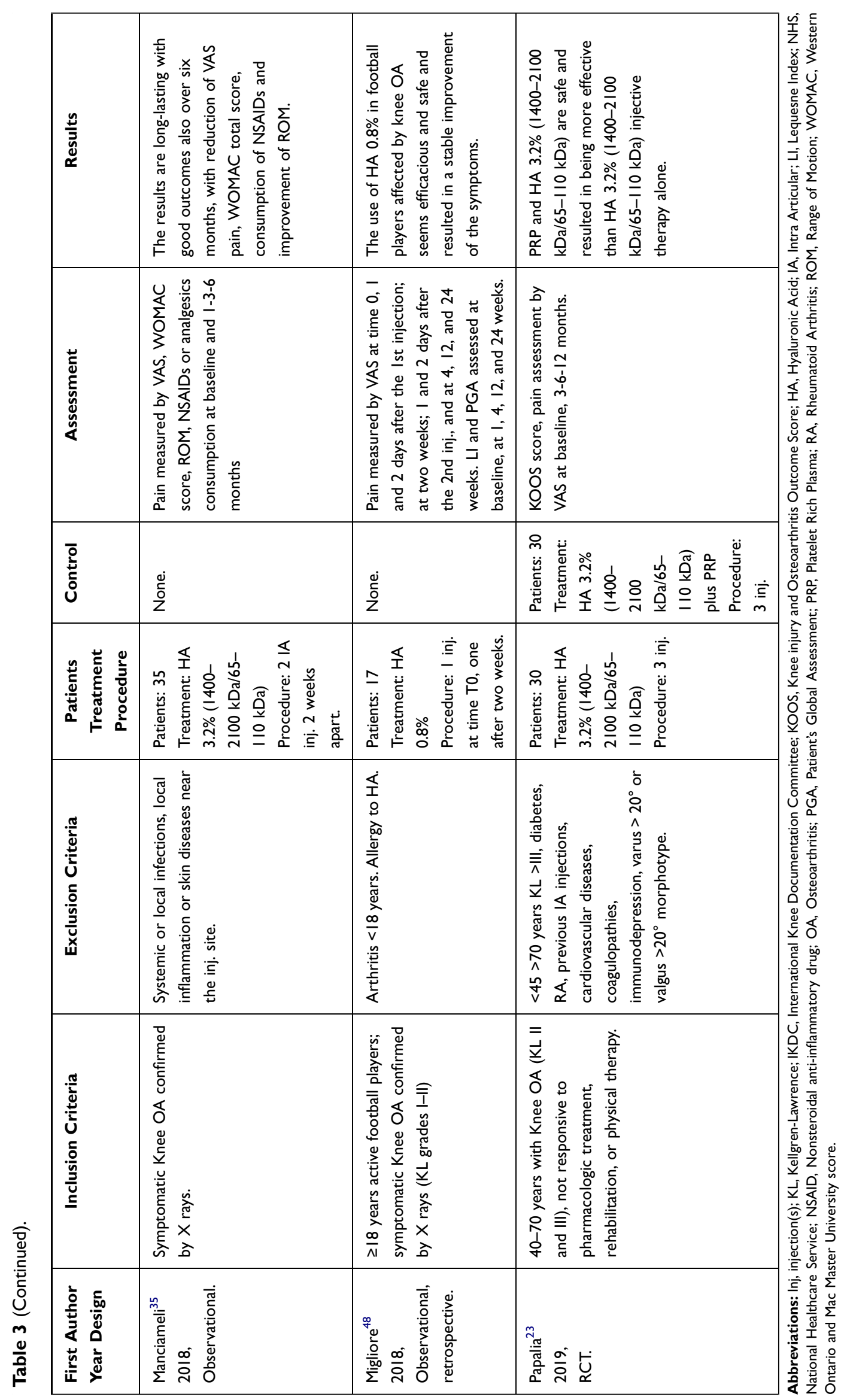




\begin{tabular}{|c|c|c|c|}
\hline 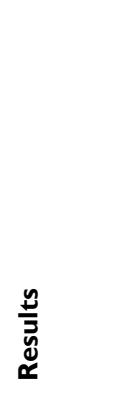 & 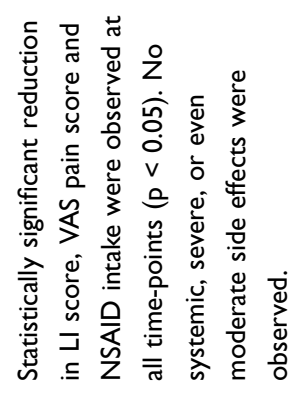 & 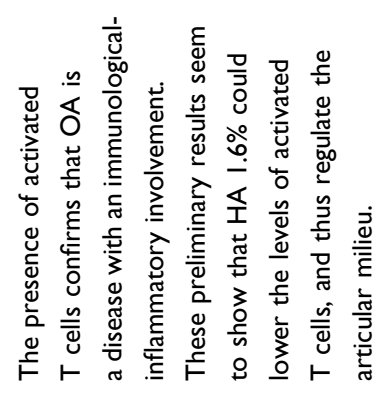 & 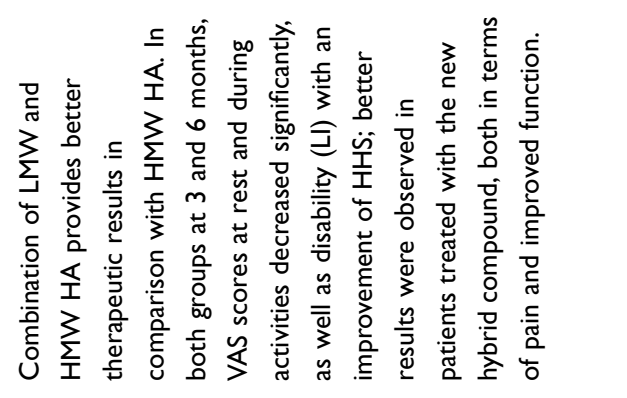 \\
\hline 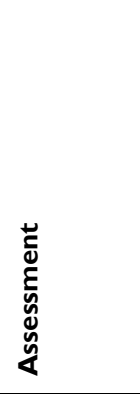 & 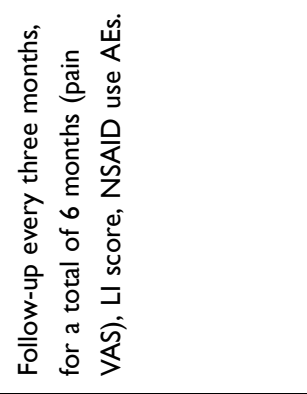 & 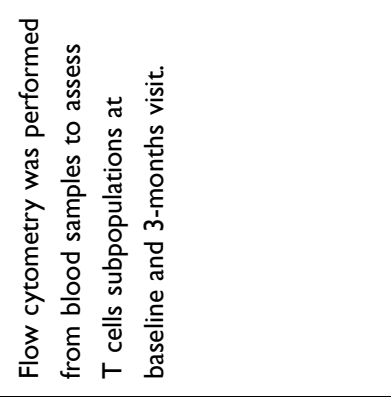 & 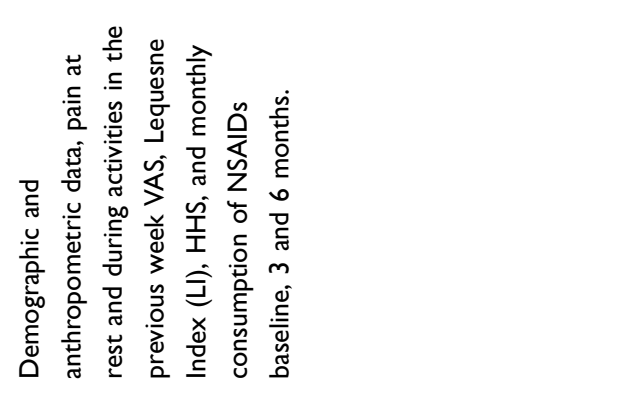 \\
\hline 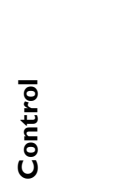 & $\begin{array}{l}\dot{j} \\
\dot{\tilde{o}} \\
\text { ż }\end{array}$ & 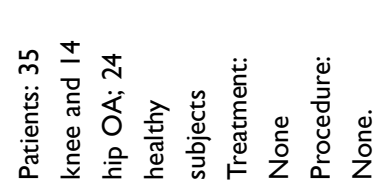 & 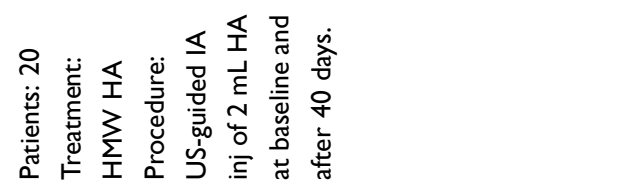 \\
\hline 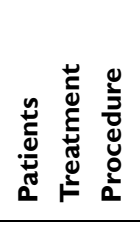 & 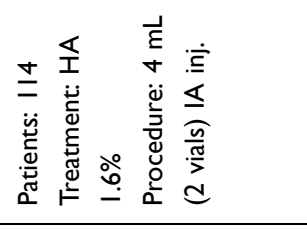 & 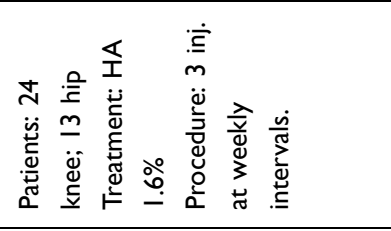 & 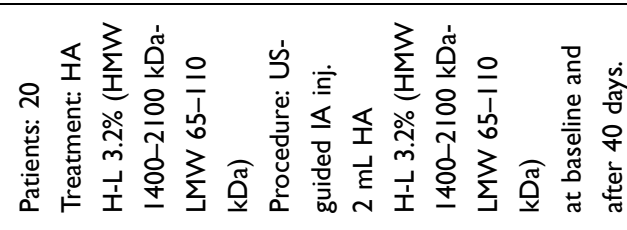 \\
\hline 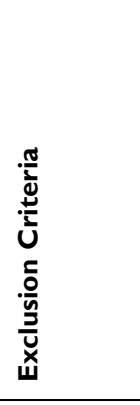 & 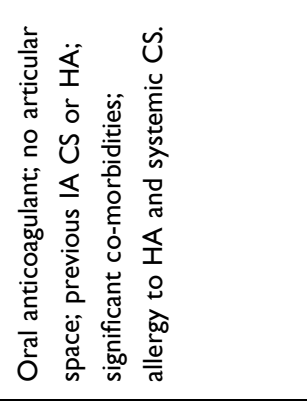 & 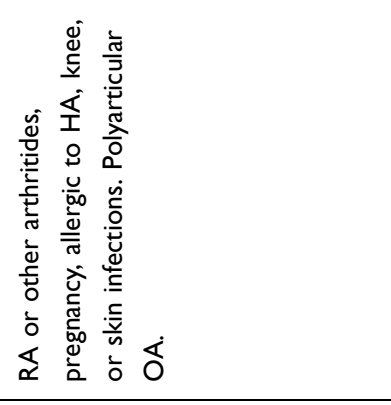 & 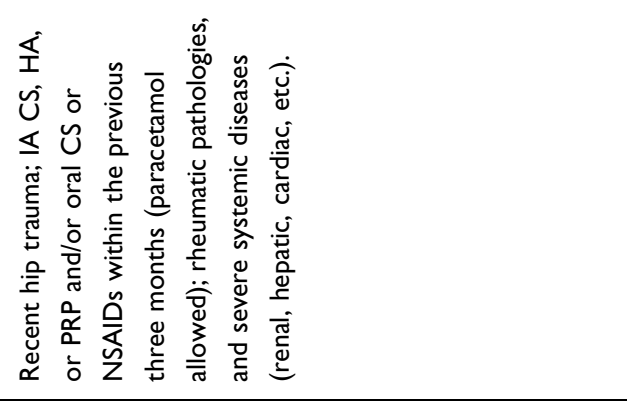 \\
\hline 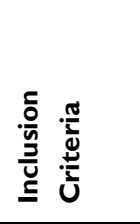 & 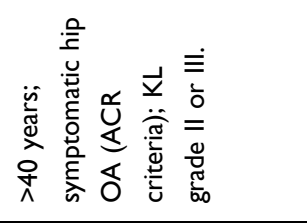 & 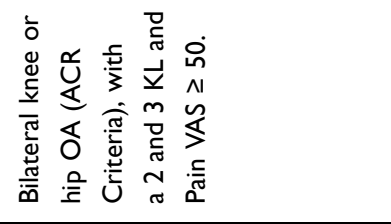 & 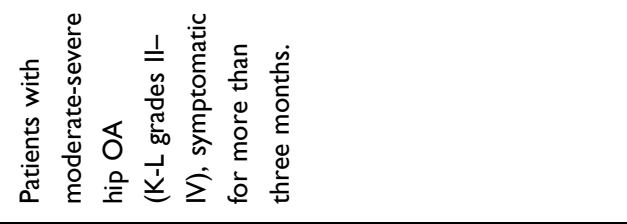 \\
\hline 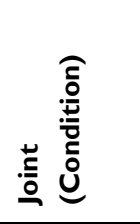 & $\begin{array}{l}\dot{\widehat{\delta}} \\
\text { O } \\
\text { 言 }\end{array}$ & 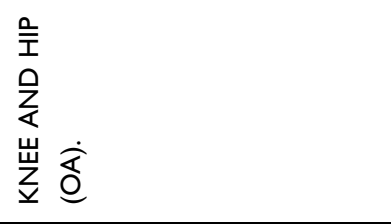 & $\begin{array}{l}\dot{\bar{\delta}} \\
\underline{0} \\
\frac{0}{\bar{I}}\end{array}$ \\
\hline 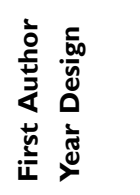 & 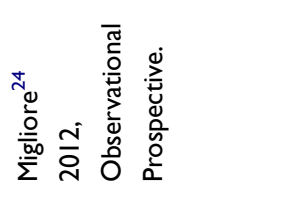 & 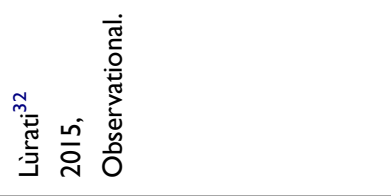 & 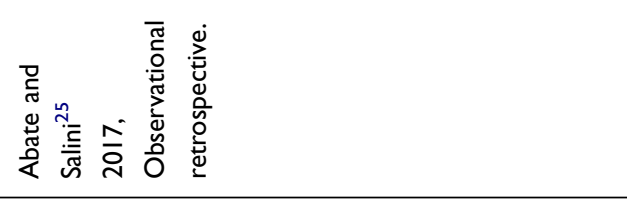 \\
\hline
\end{tabular}




\begin{tabular}{|c|c|c|}
\hline 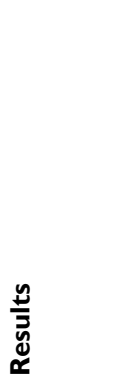 & 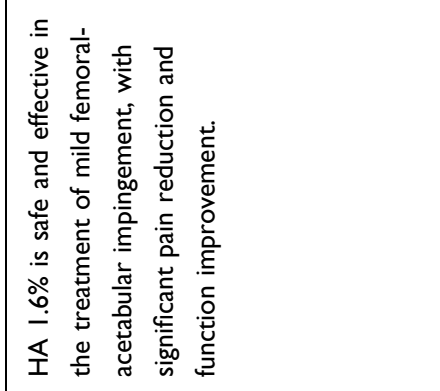 & 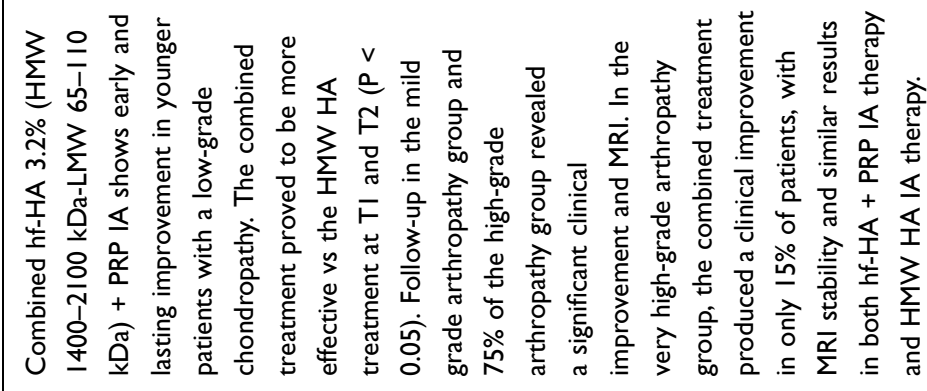 \\
\hline & 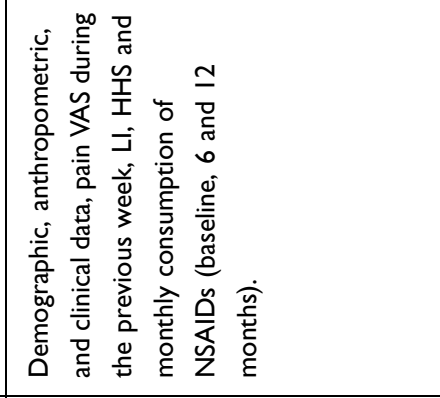 & 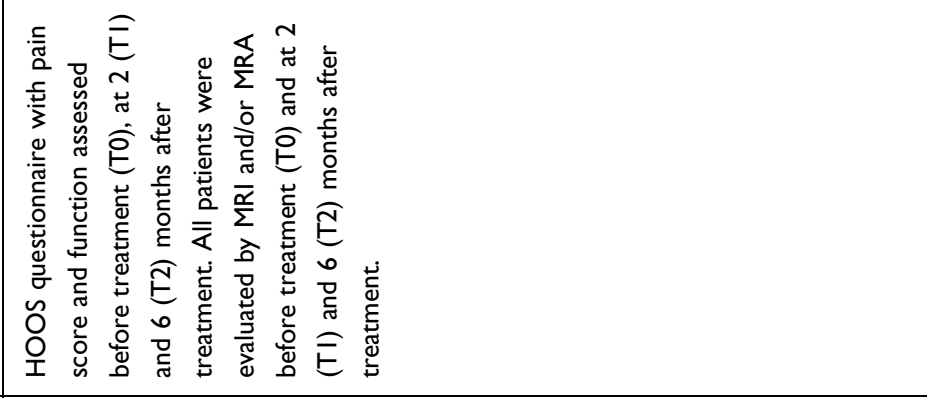 \\
\hline & $\begin{array}{l}\dot{0} \\
\stackrel{0}{0} \\
\text { z }\end{array}$ & 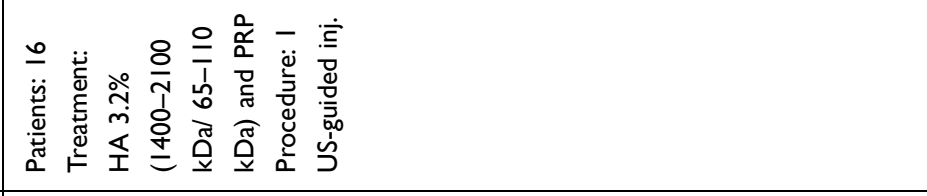 \\
\hline 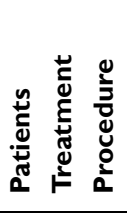 & 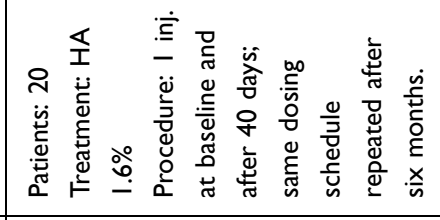 & 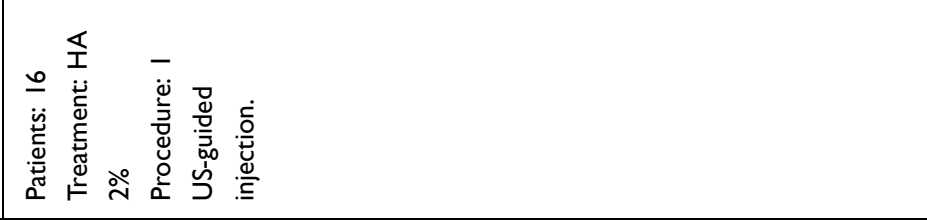 \\
\hline 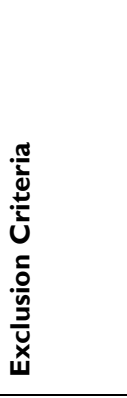 & 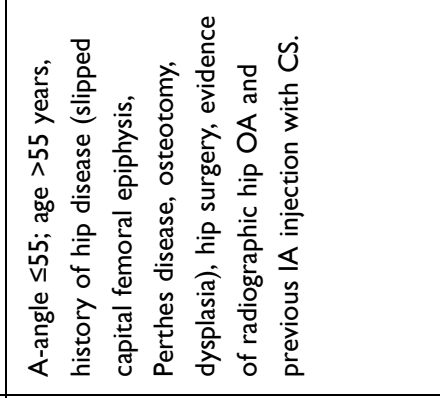 & 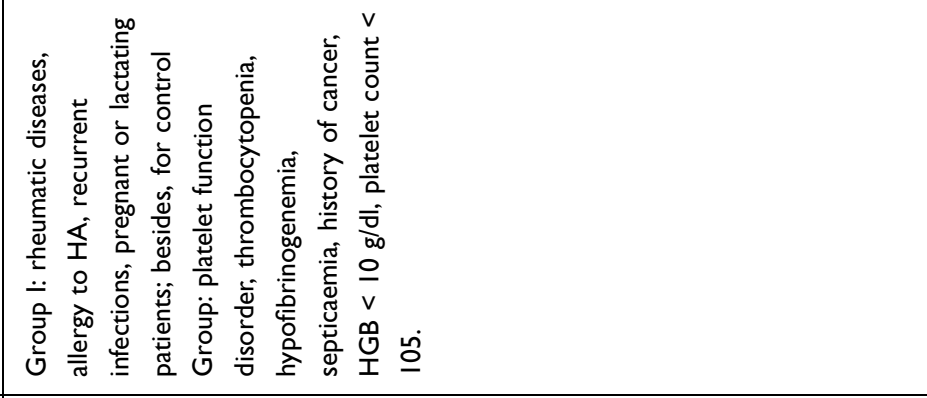 \\
\hline 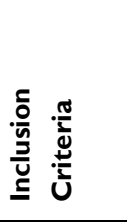 & 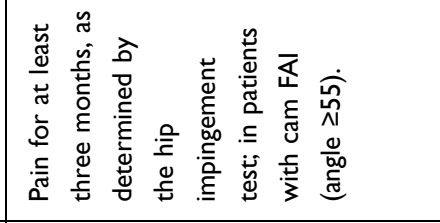 & 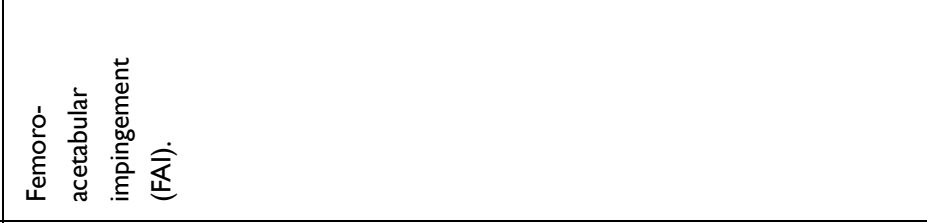 \\
\hline 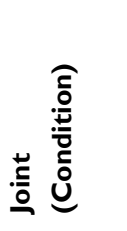 & 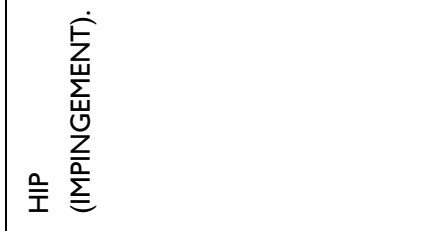 & 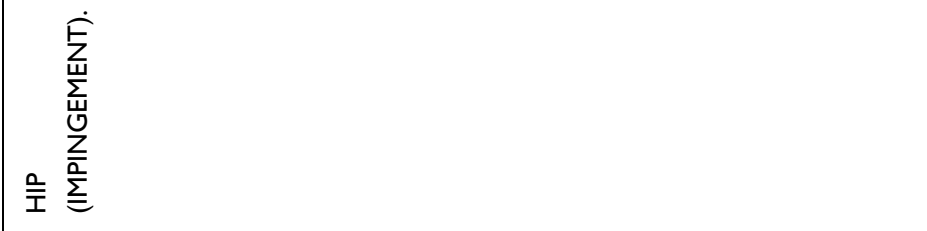 \\
\hline 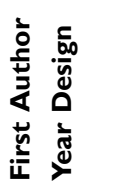 & 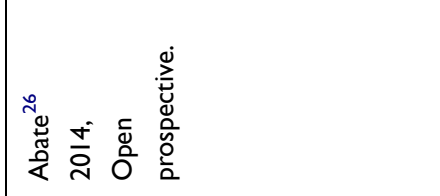 & 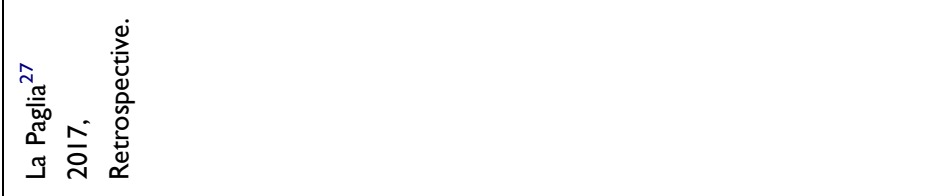 \\
\hline
\end{tabular}




\begin{tabular}{|c|c|c|}
\hline 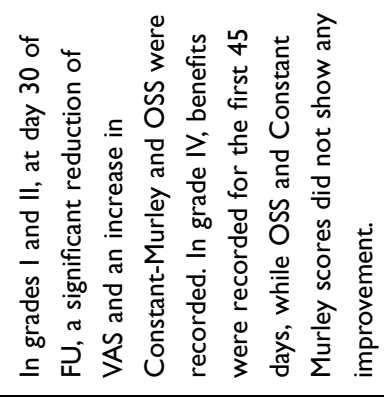 & 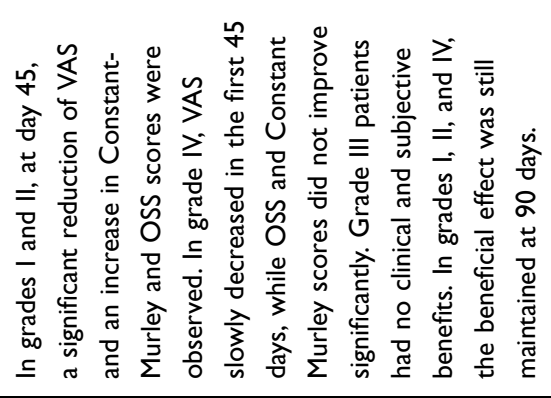 & 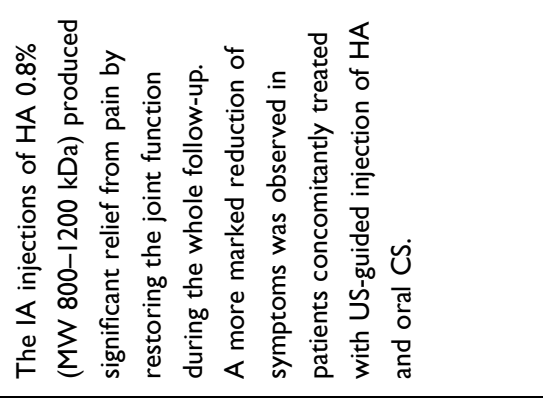 \\
\hline 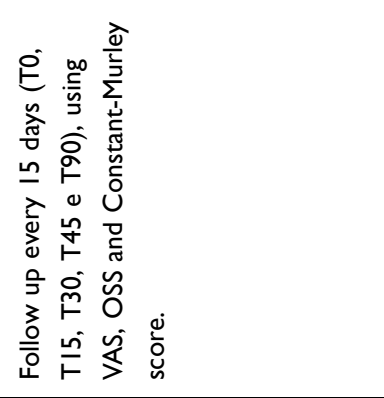 & 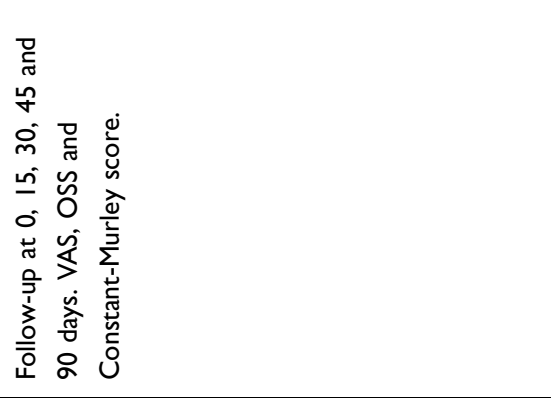 & 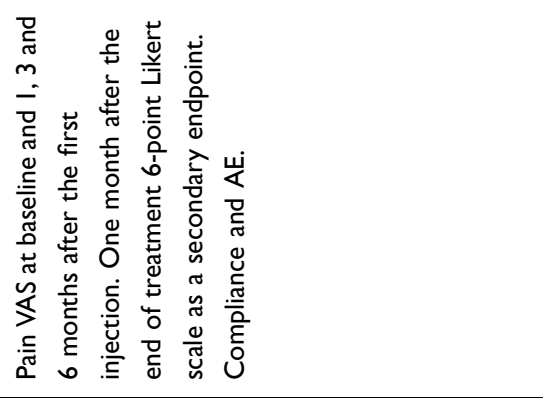 \\
\hline $\begin{array}{l}\dot{0} \\
\text { ¿े } \\
\text { z }\end{array}$ & $\begin{array}{l}\dot{0} \\
\text { ó }\end{array}$ & 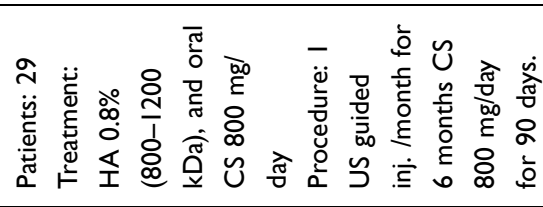 \\
\hline 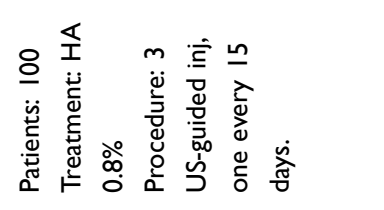 & 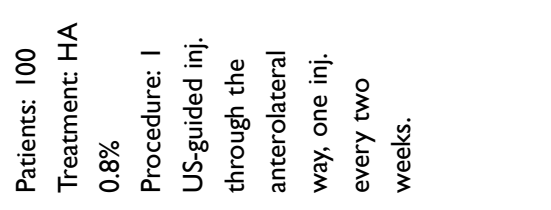 & 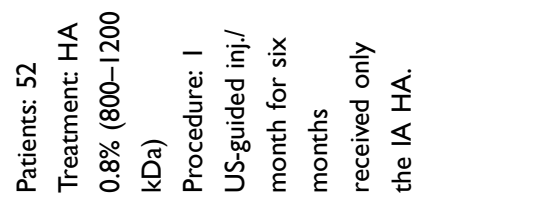 \\
\hline 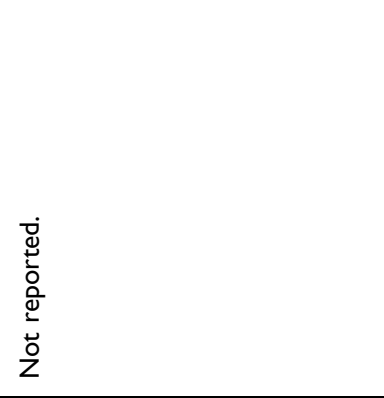 & 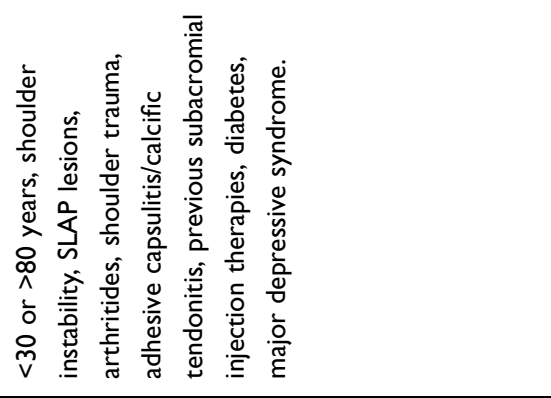 & 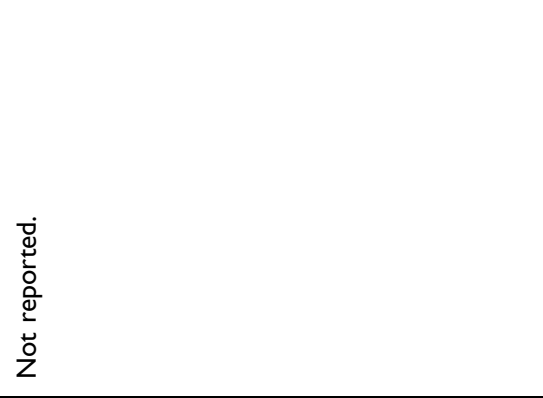 \\
\hline 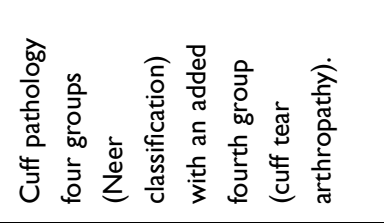 & 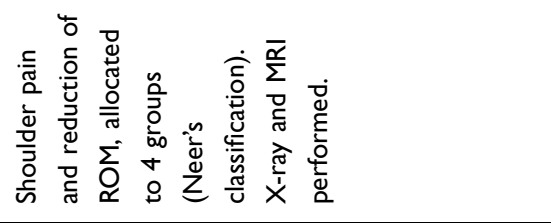 & 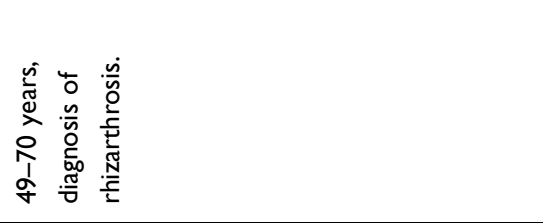 \\
\hline 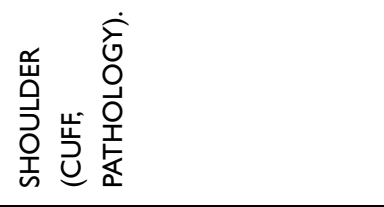 & 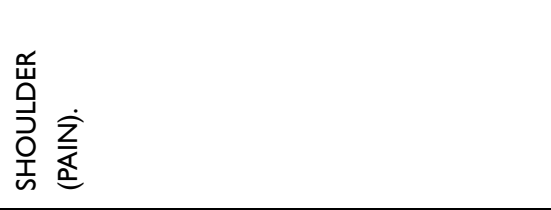 & 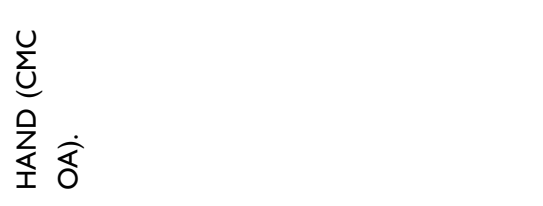 \\
\hline 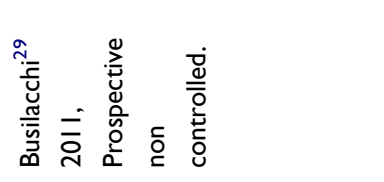 & 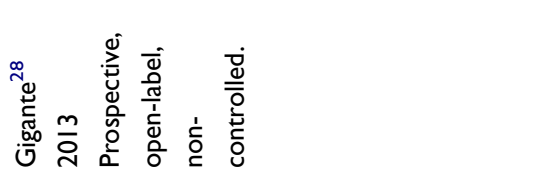 & 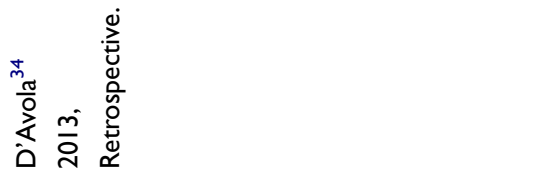 \\
\hline
\end{tabular}




\begin{tabular}{|c|c|c|c|}
\hline $\begin{array}{l}\frac{\tilde{y}}{\vec{J}} \\
\underline{\bar{y}} \\
\ddot{x}\end{array}$ & 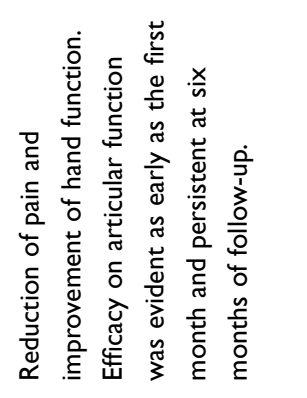 & 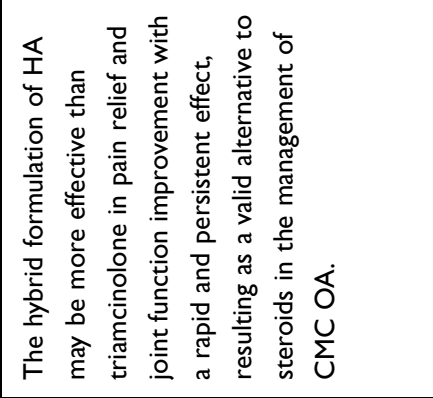 & 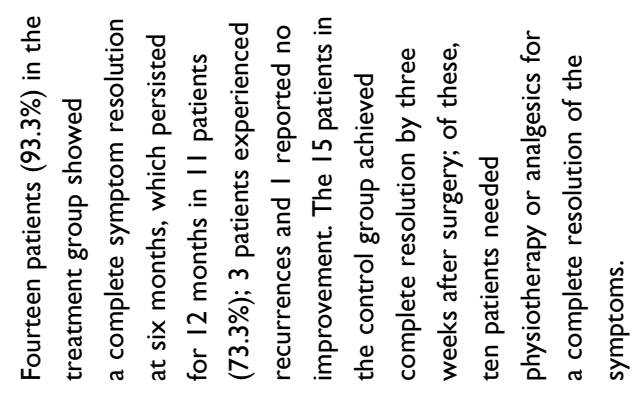 \\
\hline & 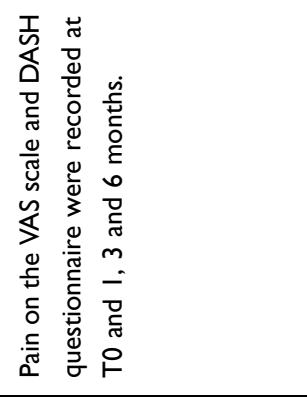 & 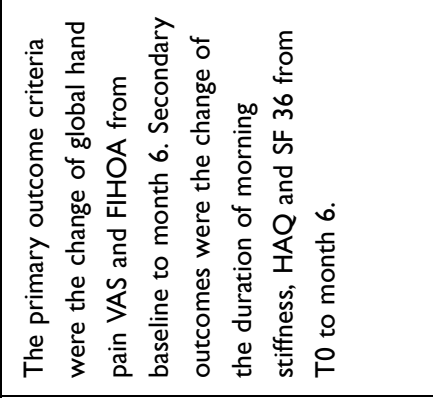 & 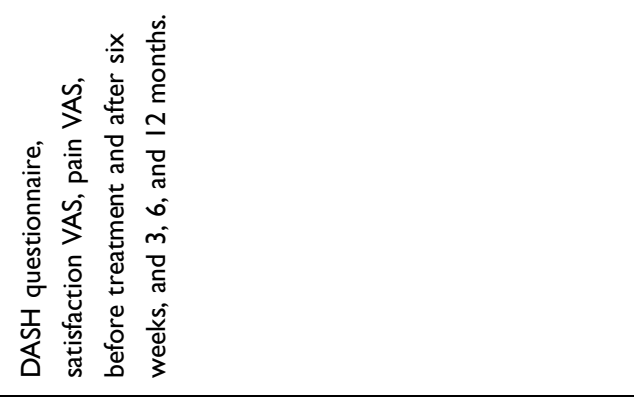 \\
\hline & $\begin{array}{l}\dot{0} \\
\text { ¿̊ } \\
\text { z }\end{array}$ & 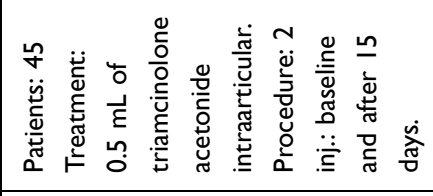 & 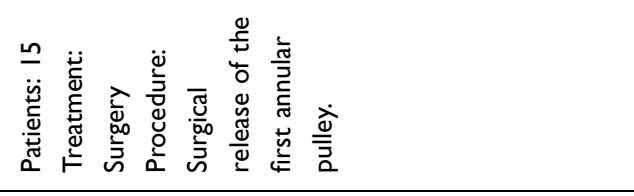 \\
\hline 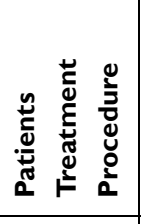 & 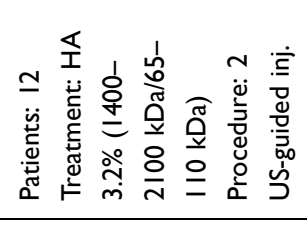 & 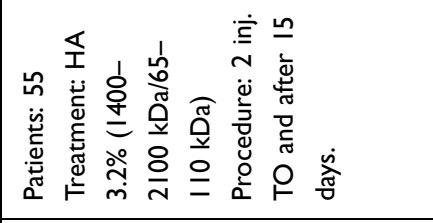 & 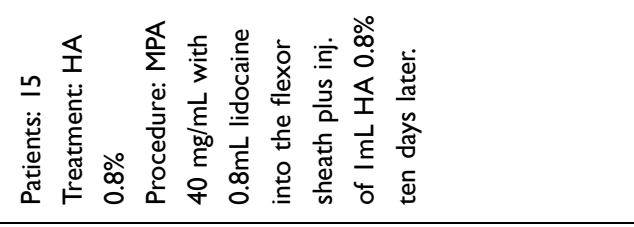 \\
\hline 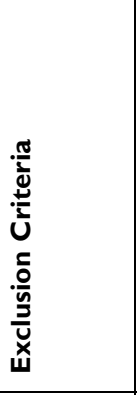 & 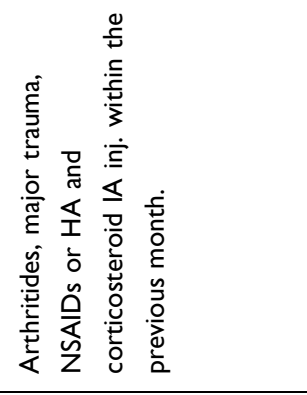 & 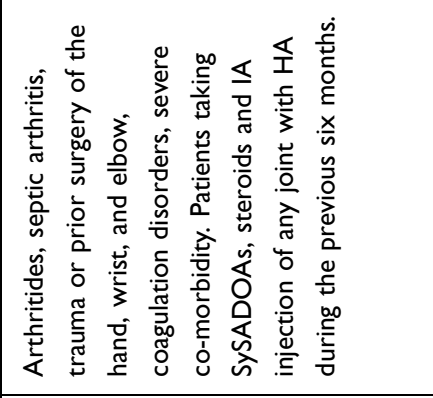 & 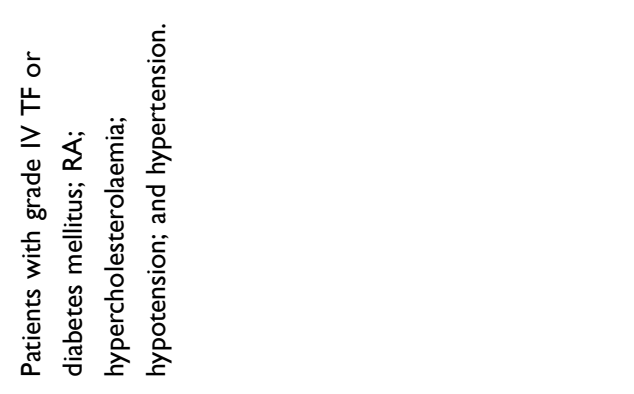 \\
\hline 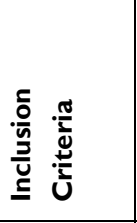 & 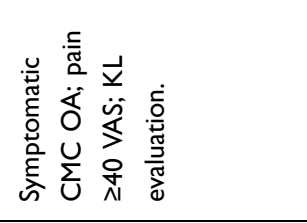 & 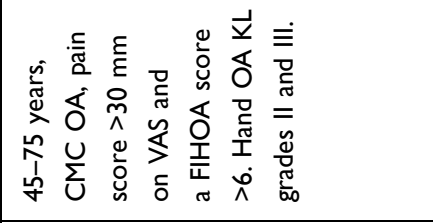 & 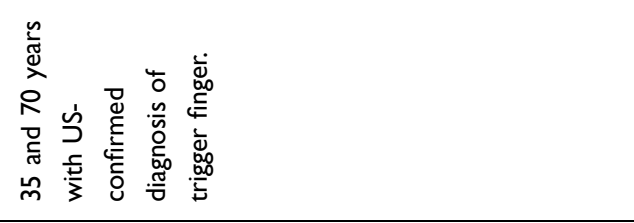 \\
\hline 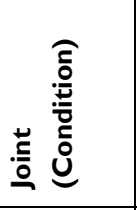 & 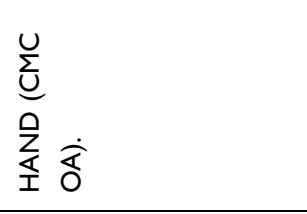 & $\begin{array}{l}\dot{0} \\
\sum \\
\underline{U} \\
0 \\
z \\
\text { I }\end{array}$ & 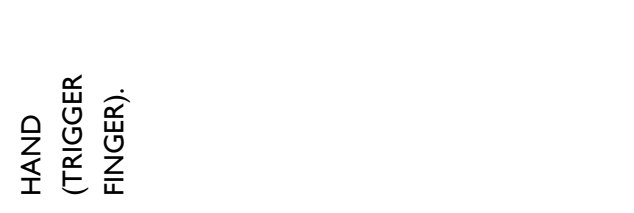 \\
\hline 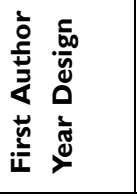 & 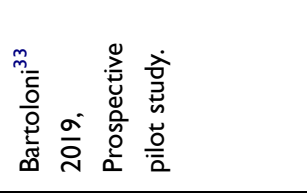 & 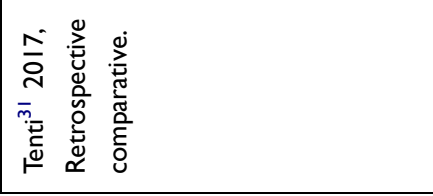 & 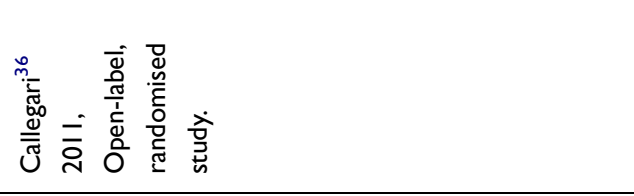 \\
\hline
\end{tabular}




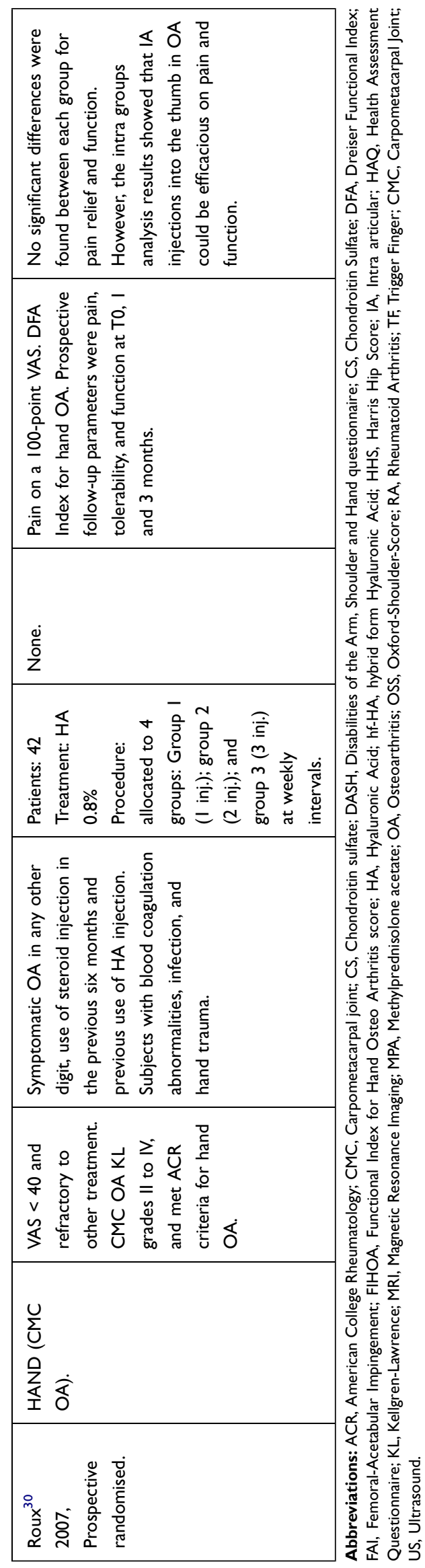

classification without indication of age. For hand localizations, Roux ${ }^{30}$ set as inclusion criteria for his study on the carpal metacarpal joint (CMC) a pain score $<40 \mathrm{~mm}$ measured by a $0-100 \mathrm{~mm}$ VAS and lack of response to other treatments. Tenti ${ }^{31}$ included patients aged between 45 and 75 years of age, with hand pain scores $>30 \mathrm{~mm}$ (VAS $100 \mathrm{~mm}$ ), with Functional Index for Hand OA (FIHOA) $>6$ and OA of KL grades II-III.

\section{Exclusion Criteria}

For hip OA, in a large cohort study, ${ }^{24}$ patients with KL grade IV, systemic co-morbidities (rheumatological disease, low back pain, femoral head osteonecrosis), obliteration of intra-articular space, previous intake of oral steroids, VS with HA, or steroids in the hip were excluded. In a retrospective observational study, Lùrati ${ }^{32}$ excluded patients with rheumatoid arthritis or other rheumatic diseases, current knee infection or skin infection around the injection site. Abate and Salini ${ }^{25}$ excluded patients with recent hip trauma, rheumatic or other severe systemic diseases, IAHA, steroids or PRP. Low back pain was an exclusion criterion in different studies. ${ }^{24,25,32}$ In hip impingement, Abate ${ }^{26}$ excluded patients with other hip pathologies, hip surgery or IA treatments. La Paglia ${ }^{27}$ compared HA $2 \%$ alone to the combination of HA 3.2\% (1400-2100 $\mathrm{kDa} / 65-110 \mathrm{kDa}$ ) and PRP. The exclusion criteria for HA were rheumatic diseases, history of allergy to HA, recurrent systemic or local infections, pregnancy, or lactation. For the patients treated with HA 3.2\% (1400-2100 $\mathrm{kDa} / 65-110 \mathrm{kDa}$ ) and PRP, additional exclusion criteria were platelet or coagulation disorders, anemia, septicemia, and previous malignancy.

For shoulder OA, in his study on pain, Gigante ${ }^{28}$ excluded patients under 30 and over 80 years of age with shoulder instability, Superior Labral Tear from Anterior to Posterior (SLAP) lesions; rheumatoid arthritis, amyloidosis, chondrocalcinosis, gout, rheumatological diseases; shoulder trauma within the last year; adhesive capsulitis/ calcific tendonitis, previous subacromial injections; diabetes, depression.

For hand OA (CMC), in an RCT comparing HA 3.2\% $(1400-2100 \mathrm{kDa} / 65-110 \mathrm{kDa})$ with IA steroids, Tenti ${ }^{31}$ excluded patients with a history of any inflammatory joint disease, septic arthritis, major trauma or prior surgery of the hand, wrist and elbow, coagulation disorders, severe co-morbidity and those who underwent therapy with chondroitin sulfate, glucosamine, diacerein, steroids by any 
route of administration and IA injection of any joint with corticosteroids or HA during the previous six months.

\section{Statements to Be Discussed}

Considering the results of the SLR, the steering committee proposed 19 statements and three assessment domains. During the first TEP meeting, the scientific evidence retrieved was presented and discussed. At the end of the meeting, the TEP defined a questionnaire composed of 25 statements grouped into three domains.

The questionnaire was discussed in two rounds of Delphi survey to find an agreement among the participants. The agreement with each statement was defined according to the RAND/UCLA criteria, with a vote from 1 (total disagreement) to 9 (total agreement). The agreement and disagreement among the experts were defined as follows: agreement if $80 \%$ of the 14 panelists' ratings were concentrated in one of the 3-point regions $(1-3 ; 4-$ 6 ; 7-9); disagreement if $90 \%$ of the 14 panelists' ratings were spread across one of the two extra-wide regions (1-6 or 4-9).

The whole process was carried out using a web platform. The results of the questionnaire were discussed by the TEP during the second plenary meeting. Unanimity was reached in the domains, and 21 items (Table 5) were selected.

\section{Patients' Features: Characteristics of the Patient to Be Considered Before Planning the VS}

The TEP agreed that VS provides the best results in patients with primary and secondary knee and hip OA of KL grades I to III. The TEP pointed out the importance of considering that the efficacy of VS is influenced not only by the severity of the condition (eg KL grade) but also by the phenotype of the patient. Patients with OA of KL grades II and III without malalignment or dissymmetry and with BMI < 30 can be successfully treated; on the other hand, in case of severe malalignment of the extremity, instability in the affected joint and meniscal lesion, the outcome of VS could be less favorable.

The TEP also agreed on further advice concerning specific localizations:

Knee: The TEP considered that VS is possible in patients with knee OA of KL grade IV in selected cases: i) persistent, localized knee pain ii) $\mathrm{BMI}<30$ iii) walking limitation iv) pain when squatting v) patients who failed to respond sufficiently to analgesics and/or NSAIDs taken regularly vi) patients who responded well but who were unable to tolerate analgesics and/or NSAIDs vii) absence of malalignment viii) absence of lower limbs dissymmetry.

Hip: Patients with hip OA of KL grades II, III, without malalignment, lower limbs dis-symmetry or with BMI < 30 could be treated. The TEP recommended excluding patients with hip OA of KL grade IV confirmed by X-rays, overweight $(\mathrm{BMI}>30)$, with malalignment or lower limb dis-symmetry.

CMC: The TEP concluded that patients with CMC OA of KL grades I-II-III can be treated. VS with HA can be considered in patients with CMC OA of KL grade IV confirmed by X-rays only in selected cases: patients aged between 50 and 75 years with clinical manifestations for more than three months, defined as global hand pain score more than $30 \mathrm{~mm}$ on a $0-100$ VAS and a Functional Index for Hand OA (FIHOA) score $>6$. $^{31,33,34}$

\section{Conditions That are Linked to Increased Risk of Failure}

The TEP discussed cases in which VS is contraindicated, or there may be an increased risk of failure. VS with HA and other HA preparations is contraindicated in case of hypersensitivity to HA, in systemic septic conditions, in cutaneous diseases or infection in the area surrounding the injection site. VS is not indicated in patients assuming double antiaggregant or New Oral Anti Coagulants (NOACs). However, some clinicians suggest that during treatment with NOACs, the injection in the knee is possible in the hands of a skilled, senior physician. VS is also contraindicated if the joint is affected by severe inflammation, active or occurring in the previous 3-6 months. Other conditions in which VS is not indicated are active inflammatory arthropathies (rheumatoid arthritis, psoriatic arthritis) and crystal arthropathies (chondrocalcinosis, gout, etc.), accompanied by synovitis. After reviewing the evidence and considering the clinical experience of the panelists, the TEP agreed that VS could be performed in case of isolated femoro-patellar syndrome or severe varus/valgus deformity in the target knee. The clinician should, however, be aware that these conditions are linked to an increased risk of failure.

\section{Conditions in Which VS is Considered Appropriate}

The TEP discussed the conditions occurring in daily clinical practice in which VS with $\mathrm{HA}$ can be considered appropriate, ie conditions when VS provides specific advantages to patients. This is because, in RCTs, the study population consists of "ideal" patients, without co- 
Table 5 Domains and Items Approved After Discussion

\begin{tabular}{|c|c|c|c|c|}
\hline \multirow[t]{2}{*}{ Domain } & \multirow[t]{2}{*}{ Item } & \multicolumn{3}{|c|}{ Aggregation (\%) } \\
\hline & & 7-9 & 4-6 & $1-3$ \\
\hline $\mathbf{I}$ & Patients features. Characteristics of the patient to be considered before starting the VS. & & & \\
\hline I.I & Patients affected by primary OA can be a candidate to VS with HA & 100 & 0 & 0 \\
\hline 1.2 & Patients affected by secondary OA can be a candidate to VS with HA & 100 & 0 & 0 \\
\hline 1.3 & $\begin{array}{l}\text { Patients with X-ray KL grades I-II-III are the better categories of patients affected by OA of the knee to treat } \\
\text { with HA }\end{array}$ & 92.9 & 7.1 & 0 \\
\hline 1.4 & In patients with X-ray KL grade IV affected by knee OA HA can be used in selected cases. & 85.7 & 14.3 & 0 \\
\hline 1.5 & Patients with X-ray KL grades I-II-III hip OA are the most suitable patients for VS with HA & 85.7 & 14.3 & 0 \\
\hline 1.6 & Patients affected by $\mathrm{OA}$ of the CMC with $\mathrm{X}$-ray $\mathrm{KL}$ grades I-II-III-IV can be treated with HA & 71.4 & 28.6 & 0 \\
\hline 2 & Risk of failure (Conditions and contraindications that are linked to increased risk of failure) & & & \\
\hline 2.1 & An isolated femoral patellar syndrome at the target knee is a condition linked to increased risk of failure. & 64.3 & 28.6 & 7.1 \\
\hline 2.2 & Severe varus/valgus deformity in the target knee is a condition linked to increased risk of failure. & 71.4 & 21.4 & 7.1 \\
\hline 2.3 & $\begin{array}{l}\text { Viscosupplementation with HA is not indicated in active arthritides with acute synovitis (rheumatoid arthritis, } \\
\text { ankylosing spondylitis, psoriatic arthritis, etc.). }\end{array}$ & 85.7 & 14.3 & 0 \\
\hline 2.4 & $\begin{array}{l}\text { Viscosupplementation with HA is not indicated in crystal arthropathies with acute synovitis (eg } \\
\text { chondrocalcinosis, gout, etc.). }\end{array}$ & 100 & 0 & 0 \\
\hline 2.5 & Viscosupplementation with HA is not indicated in patients with active systemic sepsis. & 100 & 0 & 0 \\
\hline 2.6 & $\begin{array}{l}\text { Viscosupplementation with HA is contraindicated in patients with septic knee arthritis within the previous 3-6 } \\
\text { months. }\end{array}$ & 92.9 & 7.1 & 0 \\
\hline 2.7 & Viscosupplementation with $\mathrm{HA}$ is contraindicated in patients with hypersensitivity to HA. & 92.9 & 7.1 & 0 \\
\hline 2.8 & Viscosupplementation with HA is contraindicated in the presence of an infected or seriously inflamed joint. & 100 & 0 & 0 \\
\hline 2.9 & $\begin{array}{l}\text { Viscosupplementation with HA is contraindicated in patients with cutaneous disease or infection in the area of } \\
\text { the injection site. }\end{array}$ & 92.9 & 7.1 & 0 \\
\hline 2.10 & $\begin{array}{l}\text { Viscosupplementation with HA is not indicated in patients assuming double antiaggregant and NOACs. With } \\
\text { NOACs, the injection in the knee is possible if performed by a skilled, senior doctor. }\end{array}$ & 57.1 & 28.6 & 14.3 \\
\hline 3 & Clinical situations in which the VS with HA is considered appropriate & & & \\
\hline 3.1 & The use of HA in patients taking systemic NSAIDs is recommended to reduce the assumption of these drugs. & 92.9 & 7.1 & 0 \\
\hline 3.2 & $\begin{array}{l}\text { The concomitant use of HA in patients taking SySADOAs (chondroitin sulfate, glucosamine, etc.) may improve } \\
\text { clinical efficacy. }\end{array}$ & 92.9 & 0 & 7.1 \\
\hline 3.3 & $\begin{array}{l}\text { A treatment model associating intra-articular HA to exercise and rehabilitative interventions can be useful to } \\
\text { improve joint function. }\end{array}$ & 92.9 & 0 & 7.1 \\
\hline 3.4 & $\begin{array}{l}\text { In the case of multiple joint involvement, } \mathrm{HA} \text { can be considered in different joints (at best step by step starting } \\
\text { from the most painful joint). }\end{array}$ & 85.7 & 7.1 & 7.1 \\
\hline 3.5 & $\begin{array}{l}\text { The injection of HA after a failure of other viscosupplements, having significantly different characteristics from } \\
\text { HA (MW, concentration, and volume), can be given to verify if the change of product is associated to a better } \\
\text { clinical response. }\end{array}$ & 85.7 & 14.3 & 0 \\
\hline
\end{tabular}

Abbreviations: HA, hyaluronic acid; KL, Kellgren-Lawrence; MW, Molecular Weight; NOAC, Novel Anti Coagulant; NSAID, Nonsteroidal Anti-inflammatory drug; OA, Osteoarthritis; CMC, Carpometacarpal Joint; SySADOA, Symptomatic Slow Acting Drugs for Osteoarthritis; VS, Viscosupplementation. 
morbidities and concomitant treatments that are common in a real-life setting. Besides, because of the chronicity of the disease, the treatment of real-life patients is longer than that of patients enrolled in RCTs and requires integration or shadowing with other treatments. The TEP considered first that the association of VS, exercise and rehabilitative interventions could be useful to improve joint function. Moreover, VS is a means to reduce the intake of NSAIDs. ${ }^{25,36}$ VS can be performed in conjunction with the use of SySADOAs to obtain a synergistic action. In this regard, the TEP considered is of particular interest the study by D'Avola ${ }^{34}$ in which 52 patients received HA $0.8 \%(800-1200 \mathrm{kDa})$, and 29 received HA $0.8 \%$ (800$1200 \mathrm{kDa}$ ) plus chondroitin sulfate P.O. A more marked reduction of pain (VAS from 70 to 10 at three months: $\mathrm{p}<0.01$ and at six months: $\mathrm{p}<0.02$ ) was observed in patients treated with HA $0.8 \%(800-1200 \mathrm{kDa})$ and chondroitin sulfate P.O. As the effect of VS becomes evident after a few weeks, while the SySADOAs need a few months before showing an evident clinical effect, the combination of VS and SySADOAs may not be effective in the acute phase. In case of multiple localizations of OA, VS can be performed in multiple target joints (especially bilateral injection in the knee is feasible) when OA is of initial or intermediate grade, while in other cases the concomitant administration of SySADOAs or NSAIDs could be considered. Finally, VS with HA after the failure of other viscosupplements having significantly different characteristics (MW, concentration, and volume) can be tried to verify if the change of product could be associated to a better clinical response.

\section{Items in Disagreement}

The TEP discussed conditions in which further research was deemed opportune.

In hip impingement, $\mathrm{Abate}^{26}$ found that VS with HA $1.6 \%$ significantly reduced $(\mathrm{p}<0.0001)$ the pain measured by VAS, the Lequesne Index (LI) and the Harris Hip Score (HSS) at 6 and 12 months. A retrospective open study conducted by La Paglia ${ }^{27}$ compared the efficacy of HA $2 \%$ with a combination of HA $3.2 \%(1400-2100 \mathrm{kDa} / 65-110 \mathrm{kDa})$ and PRP using imaging and a Hip disability and Osteoarthritis Outcome Score (HOOS) questionnaire exploring pain and function at baseline, 2 and 6 months after treatment. The Authors found that the combination of HA $3.2 \%(1400-2100 \mathrm{kDa} / 65-110 \mathrm{kDa})$ and PRP was more effective than HA $2 \%$ alone in the reduction of HOOS and at the MRI imaging. The TEP concluded that this preliminary evidence required further investigation. In Shoulder localization, VS with HA $0.8 \%$ in 100 patients affected by cuff pathology (grades I-IV of Neer's classification) induced reduction of pain measured by VAS at 30 days, as well as improvement of the OSS and Constant-Murley C-M score, the treatment being more effective in grades I and II. $^{29}$ Similar results were obtained by Gigante: ${ }^{28}$ in patients with OA of grades I and II, HA $0.8 \%$ reduced the pain measured by VAS and improved the OSS and the C-M score starting from 15 and up to 90 days. VS proved to be less effective in grades III and IV. The TEP concluded that further studies are needed to assess the efficacy of HA $0.8 \%$ and its indication in the treatment of rotator cuff syndrome, shoulder OA, impingement, and pain.

Stenosing tenosynovitis (Trigger finger): Callegari $^{36}$ compared HA $0.8 \%+$ prednisolone and lidocaine (15 patients) with open surgery (15 patients) in the treatment of trigger finger. The patients were assessed before the procedure and at 6 weeks, 3, 6 and 12 months after the procedure. At 12 months, VS had similar efficacy and lower overall burden (time to recovery, care of the surgical wound) on the patient. However, due to the limited number of patients, the TEP considered further research on the subject necessary.

\section{Research Agenda}

The statement on which agreement could not be reached would form a "research agenda", defining topics of interest in future research.

The TEP considered interesting to design future research to assess the efficacy of VS in the prevention of the progression of OA from its early stage; the prospective collection of morphological data could also allow a better understanding of the efficacy of the combination of VS with oral SySADOAs in limiting or slowing the structural progression of OA. A second topic that the panelists also considered worthy of further research is the assessment of the value of imaging (MRI, US, etc.) in the detection of patients most suitable for treatment with VS and in the definition of patients in which VS has the highest possibility of success. More information is needed on Grade IV CMC OA and ankle OA. Further research should be conducted to assess the efficacy of VS in hemophilia and postacute chondrocalcinosis. Finally, the TEP agreed on the importance of studying phenotypes that are more suitable for each specific HA formulation since, to date, the choice of the formulation to be used is based on the experience of the operator. 


\section{Discussion}

Among the strengths of this review is the multidisciplinary team that carried over the review. Moreover, a rigorous and well-codified methodology was used for the collection and appraisal of the clinical evidence. MMAT revealed an overall good quality of the selected papers.

As for the limitations of the review, one is the focus on a single product. As discussed above, this choice met two criteria: feasibility, to allow an in-depth analysis of each paper and homogeneity, concentrating the analysis on the medical condition itself and not on the comparison of different products. The second limitation of the review is that the TEP did not include representatives of patients and General Practitioners (GPs). This is because the focus of this review was on the VS technique, yet the TEP considered the patients' and GPs' point of view of primary importance and recommended their involvement in future assessments on VS.

Concerning the results of the review, the TEP reached an excellent level of agreement on the evaluation of available efficacy and safety data. Considering the data discussed above, the authors concluded that HA has a favorable benefit/risk ratio. This evaluation was consistent with the results of a previous review on HA. ${ }^{37}$

This review cannot be considered conclusive. The field of VS is evolving, and there are many aspects to be studied as mentioned in the research agenda. First, the possibility that VS, in association with SySADOAs, could prevent the progression of OA, as hypothesized in previous reviews. ${ }^{38-40}$ Another key area of study is the role of imaging. While there is a large body of scientific evidence on the use of various imaging techniques to guide the procedure of $\mathrm{VS},{ }^{41-43}$ little is known on the possibility to use imaging techniques to select the patients most suitable for the VS. Finally, the VS in specific medical conditions is still controversial; it is the case of chondrocalcinosis, where IAHA is not recommended. ${ }^{44}$ Further research should focus on the identification of new therapeutic strategies based on the definition of therapeutic sequencing and/or association between VS and other IA compounds, as well as pharmacological and non-pharmacological treatments. The last field worthy of further research is the definition of types of IAHA most suitable in various clinical conditions. There are several types of IAHA, differing mainly for their molecular weight, which influences not only the rheological behavior of the HA but also the interaction of HA with the biological structures. ${ }^{45}$

\section{Conclusion}

This systematic literature review and Expert Opinion discuss studies with a sound scientific methodology. The TEP analyzed the use of VS with HA in different localizations of OA. The results of the review can be summarized as follows: i. VS is safe and effective in most cases of knee and hip OA of KL grades I to III; ii. A careful evaluation of the medical history and concomitant conditions should be conducted before undertaking the procedure iii. The evaluation of the patient should not be limited to the assessment of KL grade but should also consider concomitant conditions that could reduce the efficacy of the procedure iv. Specific locations and situations require an additional evaluation from the clinician. v. VS can be usefully complemented by rehabilitation programs and the use of SySADOAs.

\section{Acknowledgments}

Dr Salvatore Bianco of AKROS BioScience (Pomezia, Italy) provided medical writing and editing services during the preparation of the paper.

\section{Author Contributions}

All authors contributed to data analysis, drafting or revising the article, gave final approval for the version to be published, agreed to the submitted journal, and agreed to be accountable for all aspects of the work.

\section{Funding}

The publication of this article was supported by a nonconditioning grant from IBSA International (Lugano, Switzerland).

\section{Disclosure}

Dr Blicharski and Dr Matucci Cerinic report personal fees from IBSA, outside the submitted work. Dr Migliore reports fees for participation in review activities from Fidia, MSD, Sanofi and Novartis, and payment for lectures from Roche, GUNA, IBSA, Abiogen and Pfizer, outside the submitted work. Dr Bannuru reports grants and personal fees from Pfizer, outside the submitted work. Dr Chevalier reports personal fees for expert and symposium from IBSA, nonfinancial expert support for Sanofi, personal fees from Flexion Therapeutics and Macopharma, outside the submitted work. The authors report no other conflicts of interest in this work. 


\section{References}

1. Hunter DJ, Bierma-Zeinstra S. Osteoarthritis. Lancet. 2019;393 (10182):1745-1759. doi:10.1016/S0140-6736(19)30417-9

2. Roddy E, Menz HB. Foot osteoarthritis: latest evidence and developments. Ther Adv Musculoskelet Dis. 2018;10(4):91-103. doi:10.1177/1759720X17753337

3. Peyron JG, Balazs EA. Preliminary clinical assessment of Na-hyaluronate injection into human arthritic joints. Pathol Biol. 1974;22(8):731-736.

4. Nelson AE, Allen KD, Golightly YM, Goode AP, Jordan JM. A systematic review of recommendations and guidelines for the management of osteoarthritis: the chronic osteoarthritis management initiative of the U.S. bone and joint initiative. Semin Arthritis Rheum. 2014;43(6):701-712. doi:10.1016/j.semarthrit.2013.11.012

5. Bannuru RR, Osani MC, Vaysbrot EE, et al. OARSI guidelines for the non-surgical management of knee, hip, and polyarticular osteoarthritis. Osteoarthritis Cartilage. 2019;27(11):1578-1589. doi:10.1016/j.joca.2019.06.011

6. Jordan KM, Arden NK, Doherty M, et al. EULAR recommendations 2003: an evidence based approach to the management of knee osteoarthritis: report of a Task Force of the Standing Committee for International Clinical Studies Including Therapeutic Trials (ESCISIT). Ann Rheum Dis. 2003;62(12):1145-1155. doi:10.1136/ ard.2003.011742

7. National Collaborating Centre for Chronic Conditions (UK). Osteoarthritis: National Clinical Guideline for Care and Management in Adults. London: Royal College of Physicians (UK); 2008.

8. Jevsevar DS. Treatment of osteoarthritis of the knee: evidence-based guideline, 2nd edition. J Am Acad Orthop Surg. 2013;21(9):571-576. doi:10.5435/JAAOS-21-09-571

9. Alviti F, Agostini F, Conte V, Bernetti A. Linee guida ed evidenze scientifiche sulla terapia infiltrativa intra-articolare in medicina riabilitativa (Guidelines and scientific evidence on intra-articular infiltrative therapy in rehabilitation medicine). In: Santilli V, editor. Linee guida ed evidenze scientifiche in Medicina Fisica e Riabilitativa (Guidelines and scientific evidence in Physical and Rehabilitation Medicine). Sapienza University of Rome; 2017:753.

10. Migliore A, Bizzi E, Herrero-Beaumont J, Petrella RJ, Raman R, Chevalier X. The discrepancy between recommendations and clinical practice for viscosupplementation in osteoarthritis: mind the gap! Eur Rev Med Pharmacol Sci. 2015;19(7):1124-1129.

11. Maheu E, Bannuru RR, Herrero-Beaumont G, Allali F, Bard H, Migliore A. Why we should definitely include intra-articular hyaluronic acid as a therapeutic option in the management of knee osteoarthritis: results of an extensive critical literature review. Semin Arthritis Rheum. 2019;48(4):563-572. doi:10.1016/j.semarthrit.2018.06.002

12. Bruyere $\mathrm{O}$, Honvo $\mathrm{G}$, Veronese $\mathrm{N}$, et al. An updated algorithm recommendation for the management of knee osteoarthritis from the European Society for Clinical and Economic Aspects of Osteoporosis, Osteoarthritis and Musculoskeletal Diseases (ESCEO). Semin Arthritis Rheum. 2019;49(3):337-350. doi:10.1016/j.semarthrit.2019.04.008

13. Conrozier T, Monfort J, Chevalier X, et al. EUROVISCO Recommendations for Optimizing the Clinical Results of Viscosupplementation in Osteoarthritis. Cartilage. 2020;11 (1):47-59. doi:10.1177/1947603518783455

14. Hong QN, Pluye P, Fàbregues S, et al. Mixed Methods Appraisal Tool (MMAT), version 2018. Registration of Copyright (\#1148552), Canadian Intellectual Property Office, Industry Canada.

15. Moher D, Liberati A, Tetzlaff J, Altman DG, Group P. Reprintpreferred reporting items for systematic reviews and meta-analyses: the PRISMA statement. Phys Ther. 2009;89(9):873-880. doi: $10.1093 / \mathrm{ptj} / 89.9 .873$
16. Castellacci E, Polieri T. Antalgic effect and clinical tolerability of hyaluronic acid in patients with degenerative diseases of knee cartilage: an outpatient treatment survey. Drugs Exp Clin Res. 2004;30(2):67-73.

17. Theiler R, Bruhlmann P. Overall tolerability and analgesic activity of intra-articular sodium hyaluronate in the treatment of knee osteoarthritis. Curr Med Res Opin. 2005;21(11):1727-1733. doi:10.1185/030079905X65547

18. Polacco A, Beomonte Zobel B, Polacco M, et al. The effect of intraarticular hyaluronic acid (Sinovial ${ }^{\mathbb{R}}$ One) on knee osteoarthritis: a Preliminary Study. Eur J Inflammation. 2013;11:847-853. doi:10.1177/1721727X1301100327

19. Papalia R, Zampogna B, Russo F, et al. Comparing hybrid hyaluronic acid with PRP in end career athletes with degenerative cartilage lesions of the knee. J Biol Regul Homeost Agents. 2016;30(4 Suppl 1):17-23,

20. Bottegoni C, Dei Giudici L, Busilacchi A, Chiurazzi E, Bencivenga R, Gigante A Homologous platelet-rich plasma versus hyaluronic acid intra-articular injection as treatment for knee osteoarthritis of elderly patients. Conference: 16th ESSKA Congress at: Amsterdam Volume: Knee Surg Sports Traumatol Arthrosc. Vol. 22; 2014:S6-S109.

21. Papalia R, Russo F, Torre G, et al. Hybrid hyaluronic acid versus high molecular weight hyaluronic acid for the treatment of osteoarthritis in obese patients. J Biol Regul Homeost Agents. 2017;31(4 Suppl 2):103-109.

22. Pavelka K, Uebelhart D. Efficacy evaluation of highly purified intra-articular hyaluronic acid (Sinovial((R))) vs hylan G-F20 (Synvisc $((\mathrm{R})))$ in the treatment of symptomatic knee osteoarthritis. A double-blind, controlled, randomized, parallel-group non-inferiority study. Osteoarthritis Cartilage. 2011;19(11):1294-1300. doi:10.1016/j. joca.2011.07.016

23. Papalia R, Zampogna B; Xix Congresso Nazionale S.I.C.O.O.P. Societa' Italiana Chirurghi Ortopedici Dell'Ospedalita' Privata A. The combined use of platelet rich plasma and hyaluronic acid: prospective results for the treatment of knee osteoarthritis. $J$ Biol Regul Homeost Agents. 2019;33(2Suppl. 1):21-28.

24. Migliore A, Massafra U, Bizzi E, Giovannangeli F, Tormenta S. Intra-articular ultrasound-guided injection of sinovial ${ }^{\circledR}$ forte $1.6 \%$ in patients affected by symptomatic hip osteoarthritis: effectiveness and safety in a large cohort of patients. Eur $J$ Inflammation. 2012;10:71-79. doi:10.1177/1721727X1201000108

25. Abate M, Salini V. Efficacy and safety study on a new compound associating low and high molecular weight hyaluronic acid in the treatment of hip osteoarthritis. Int J Immunopathol Pharmacol. 2017;30(1):89-93. doi:10.1177/0394632016689275

26. Abate M, Scuccimarra T, Vanni D, Pantalone A, Salini V. Femoroacetabular impingement: is hyaluronic acid effective? Knee Surg Sports Traumatol Arthrosc. 2014;22(4):889-892. doi:10.1007/ s00167-013-2581-1

27. La Paglia E, Barbero S, Belletti M, et al. Femoroacetabular impingement syndrome in young patients: efficacy of US-guided injections of platelet rich plasma and hybrid hyaluronic acid vs hyaluronic acid. G Ital Ortop Traumatol. 2017;43:215-226.

28. Gigante A, Cecconi S, Enea D, Cesari E, Valeri G, Busilacchi A. Effect of subacromial injections of hyaluronan on different grades of rotator cuff lesion: a prospective study. Eur $J$ Inflamm. 2013;3:777-787. doi:10.1177/1721727X1301100320

29. Busilacchi A, Cecconi S, Enea D. Effectiveness of the hyaluronic acid in the different stages of the evolutive cuff pathology: a perspective study. J Orthopaed Traumatol. 2011;12(Suppl 1): S33.

30. Roux C, Fontas E, Breuil V, Brocq O, Albert C, Euller-Ziegler L. Injection of intra-articular sodium hyaluronidate (Sinovial) into the carpometacarpal joint of the thumb (CMC1) in osteoarthritis. A prospective evaluation of efficacy. Joint Bone Spine. 2007;74 (4):368-372. doi:10.1016/j.jbspin.2006.08.008 
31. Tenti S, Pascarelli NA, Giannotti S, Galeazzi M, Giordano N, Fioravanti A. Can hybrid hyaluronic acid represent a valid approach to treat rizoarthrosis? A retrospective comparative study. $B M C$ Musculoskelet Disord. 2017;18(1):444. doi:10.1186/s12891-0171809-5

32. Lurati A, Laria A, Mazzocchi D, Re KA, Marrazza M, Scarpellini M. Effects of hyaluronic acid (HA) viscosupplementation on peripheral Th cells in knee and hip osteoarthritis. Osteoarthritis Cartilage. 2015;23(1):88-93. doi:10.1016/j.joca.2014.09.010

33. Bartoloni E, Luccioli F, La Paglia GMC, Cafaro G, Marcucci E, Gerli R. Effect of Sinovial High-Low(R) injections in trapeziometacarpal osteoarthritis. Clin Exp Rheumatol. 2019;37(1):166.

34. D'avola G, Tropea S. Eco-guided treatment with hyaluronic acid and concomitant administration of chondroitin sulfate $800 \mathrm{mg} /$ day in rizoarthrosis. Ann Rheum Dis. 2013;71:644.

35. Manciameli A, Peruzzi M. Trattamento della gonartrosi moderata mediante iniezione intrarticolare di acido ialuronico sale sodico (Treatment of moderate gonarthrosis by intrarticular injection of hyaluronic acid sodium salt). G Ital Ortop Traumatol. 2018;44:146-149.

36. Callegari L, Spano E, Bini A, Valli F, Genovese E, Fugazzola C. Ultrasound-guided injection of a corticosteroid and hyaluronic acid: a potential new approach to the treatment of trigger finger. Drugs R D. 2011;11(2):137-145. doi:10.2165/11591220-000000000-00000

37. Chevalier X, Migliore A. Safety and tolerability of intra-articular hyaluronic acid injection (Sinovial ${ }^{\circledR}$ ) in experimental and clinical practice. Eur J Inflamm. 2013;11:573-580. doi:10.1177/1721727X 1301100301

38. Migliore A, Bizzi E, Massafra U, et al. The impact of treatment with hylan G-F 20 on progression to total hip arthroplasty in patients with symptomatic hip OA: a retrospective study. Curr Med Res Opin. 2012;28(5):755-760. doi:10.1185/03007995.2011.645563

39. Henrotin Y, Raman R, Richette P, et al. Consensus statement on viscosupplementation with hyaluronic acid for the management of osteoarthritis. Semin Arthritis Rheum. 2015;45(2):140-149. doi:10.1016/j.semarthrit.2015.04.011
40. Bowman S, Awad ME, Hamrick MW, Hunter M, Fulzele S. Recent advances in hyaluronic acid based therapy for osteoarthritis. Clin Transl Med. 2018;7(1):6. doi:10.1186/s40169-017-0180-3

41. Gilliland CA, Salazar LD, Borchers JR. Ultrasound versus anatomic guidance for intra-articular and periarticular injection: a systematic review. Phys Sportsmed. 2011;39(3):121-131. doi:10.3810/psm.2011. 09.1928

42. Bossert M, Boublil D, Parisaux JM, Bozgan AM, Richelme E, Conrozier T. Imaging guidance improves the results of viscosupplementation with HANOX-M-XL in patients with ankle osteoarthritis: results of a clinical survey in 50 patients treated in daily practice. Clin Med Insights Arthritis Musculoskelet Disord. 2016;9:195-199. doi:10.4137/CMAMD.S40401

43. Pogliacomi F, Schiavi P, Paraskevopoulos A, et al. When is indicated viscosupplementation in hip osteoarthritis? Acta Biomed. 2018;90(1S):67-74. doi:10.23750/abm.v90i1-S.8000

44. Rosenthal AK, Ryan LM. Nonpharmacologic and pharmacologic management of CPP crystal arthritis and BCP arthropathy and periarticular syndromes. Rheum Dis Clin North Am. 2014;40(2):343-356. doi:10.1016/j.rdc.2014.01.010

45. Tavianatou AG, Caon I, Franchi M, Piperigkou Z, Galesso D, Karamanos NK. Hyaluronan: molecular size-dependent signaling and biological functions in inflammation and cancer. FEBS J. 2019;286(15):2883-2908. doi:10.1111/febs.14777

46. Depont F, Addra I, Lechevallier N, et al. Efficacy and tolerability of hyaluronic acid viscosupplementation with Sinovial for the treatment of knee osteoarthritis: a retrospective observational study. Conference abstract; 2004.

47. Galluccio F. Comparison of three treatment protocols with intra-articular low or intermediate molecular weight hyaluronic acid in early symptomatic knee osteoarthritis. Conference abstract; 2017.

48. Migliore A, Giannini S, Bizzi E, et al. Use of viscosupplementation for the recovery of active football players complaining of knee pain. Open Access J Sports Med. 2018;24(10):11-15. doi:10.2147/OAJSM. $\mathrm{S} 164693$
Orthopedic Research and Reviews

\section{Publish your work in this journal}

Orthopedic Research and Reviews is an international, peer-reviewed, open access journal that focusing on the patho-physiology of the musculoskeletal system, trauma, surgery and other corrective interventions to restore mobility and function. Advances in new technologies, materials, techniques and pharmacological agents are particularly welcome. The manuscript management system is completely online and includes a very quick and fair peer-review system, which is all easy to use. Visit http://www.dovepress.com/testimonials.php to read real quotes from published authors. 